\title{
Curvature Estimates for Minimal Annuli and Non-Compact Douglas-Plateau Problem
}

\author{
Yi FANG AND JENN-FANG HwANG
}

In this article we give curvature estimates for minimal annuli with convex boundary $L_{1} \cup L_{2}$ in parallel planes and apply these estimates to solve some kinds of non-compact Douglas-Plateau problem. The estimates for minimal annuli also give various necessary conditions for the existence of minimal surfaces.

\section{Introduction.}

In this article we give curvature estimates for minimal annuli with convex boundary $L_{1} \cup L_{2}$ in parallel planes and apply these estimates to solve some kinds of non-compact Douglas-Plateau problem. The estimates for minimal annuli also give various necessary conditions for the existence of minimal surfaces.

To state our results, let us fix some notations first.

Let $P_{t}=\left\{(x, y, z) \in \mathbb{R}^{3} ; z=t\right\}, S\left(t_{1}, t_{2}\right)=\left\{(x, y, z) \in \mathbb{R}^{3} ; t_{1} \leq z \leq\right.$ $\left.t_{2}\right\}$, and $S^{\prime}\left(t_{1}, t_{2}\right)=\left\{(x, y, z) \in \mathbb{R}^{3} ; t_{1} \leq y \leq t_{2}\right\}$, where $t_{1}<t_{2}$. Let $C_{R}$ be the solid cylinder $\left\{(x, y, z) \in \mathbb{R}^{3} ; x^{2}+y^{2} \leq R^{2}\right\}$.

Let $A \subset S\left(t_{1}, t_{2}\right)$ be an embedded minimal annulus such that $\partial A=$ $L_{1} \cup L_{2}$, where $L_{1} \subset P_{t_{1}}$ and $L_{2} \subset P_{t_{2}}$ are convex Jordan curves. Denote $\mathcal{P}_{\pi}$ the perpendicular projection on a plane $\pi$ perpendicular to the $x y$-plane. Let $\Omega_{\pi}:=\operatorname{Int}\left(\mathcal{P}_{\pi}(A)\right)$, the interior of $\mathcal{P}_{\pi}(A)$, and $\partial \Omega_{\pi}=\bigcup_{i=1}^{4} \Gamma_{i}^{\pi}$, where $\Gamma_{i}^{\pi}=\mathcal{P}_{\pi}\left(L_{i}\right), i=1,2$, and $\Gamma_{3}^{\pi}$ and $\Gamma_{4}^{\pi}$ are arcs connecting $\Gamma_{1}^{\pi}$ and $\Gamma_{2}^{\pi}$.

In Lemma 2.1 we estimate $\left|\Gamma_{i}\right|$, the length of $\Gamma_{i}$. We have

$$
\left|\Gamma_{1}^{\pi}\right|+\left|\Gamma_{2}^{\pi}\right| \geq\left|\Gamma_{3}^{\pi}\right|+\left|\Gamma_{4}^{\pi}\right|,
$$

and

$$
\left|\Gamma_{1}^{\pi}\right|\left|\Gamma_{2}^{\pi}\right|>\left(t_{2}-t_{1}\right)^{2}, \quad\left|\Gamma_{1}^{\pi}\right|+\left|\Gamma_{2}^{\pi}\right|>2\left(t_{2}-t_{1}\right) .
$$

Although the proof of the former is a simple imitation of a technique in [fanghwang-js], and the proof of the latter comes from elementary Euclidean 
geometry, the more accurate new estimate to $\Omega$ leads us to curvature estimates.

In Lemma 2.2 we prove that if $A \subset S\left(t_{1}, t_{2}\right) \cap S^{\prime}(-R, R)$ and $\pi$ is the $y z$-plane, then for any point $p \in \operatorname{Int}(A)$,

$$
\max \left\{\operatorname{dist}\left(\mathcal{P}_{\pi}(p), \Gamma_{3}^{\pi}\right), \operatorname{dist}\left(\mathcal{P}_{\pi}(p), \Gamma_{4}^{\pi}\right)\right\} \geq \frac{(\sqrt{2}-1) d^{2}}{R},
$$

where $d=\operatorname{dist}\left(p, \partial S\left(t_{1}, t_{2}\right)\right)$. Note that $\partial S\left(t_{1}, t_{2}\right)=P_{t_{1}} \cup P_{t_{2}}$, so if $p=$ $(x, y, z)$, then $d=\min \left\{z-t_{1}, t_{2}-z\right\}=\min \left\{\operatorname{dist}\left(p, P_{t_{1}}\right), \operatorname{dist}\left(p, P_{t_{2}}\right)\right\}$.

In Proposition 2.1, using Lemma 2.2 and by projecting on a suitable direction we prove an interior curvature estimate for $A \subset S\left(t_{1}, t_{2}\right) \cap C_{R}$, that is, there is a constant $C_{0}>0$, such that for any $p \in \operatorname{Int}(A)$, the Gaussian curvature of $p$ satisfies

$$
|K(p)| \leq \frac{C_{0} R^{2}}{d^{4}}
$$

where $d=\operatorname{dist}\left(p, \partial S\left(t_{1}, t_{2}\right)\right)$.

This curvature estimate gives immediate generalization of the existence result of Hoffman and Meeks, [fanghwang-hm9], to the continuous convex boundary case, see Theorem 2.2 .

Proposition 2.2 gives another curvature estimate. It states that if $A \subset$ $S\left(t_{1}, t_{2}\right) \cap S^{\prime}\left(t_{1}^{\prime}, t_{2}^{\prime}\right)$ is a compact minimal annulus such that $\partial A=L_{1} \cup L_{2}$, where $L_{1} \subset P_{t_{1}}$ and $L_{2} \subset P_{t_{2}}$ are $C^{2}$ convex Jordan curves, then there is a constant $C_{1}$ such that for any $p \in A$,

$$
|K(p)| \leq C_{1},
$$

where $C_{1}$ only depends on the height $t_{2}-t_{1}$, the width $t_{2}^{\prime}-t_{1}^{\prime}$, and the boundary planar curvature bound of $\partial A$.

These curvature estimates then lead to compactness theorems, Theorem 2.1 and Theorem 2.3, which state that some sequence of $\left\{A_{n}\right\}$ as in Proposition 2.1 or 2.2 has a convergent subsequence.

As applications of Theorem 2.1 and 2.3, we solve various non-compact Douglas-Plateau problems in Section 3.

Recall that the Douglas-Plateau problem for two contours is as follows: Let $L_{1}$ and $L_{2}$ be two disjoint Jordan curves in $\mathbb{R}^{3}$, find a minimal annulus $A$ such that $\partial A=L_{1} \cup L_{2}$.

Let $S_{1}$ and $S_{2}$ be area minimizing disks (when we say disks, we mean that they are homeomorphic to the unit disk in $\mathbb{C}$ ) such that $\partial S_{1}=L_{1}$, 
Curvature estimates annuli and non-compact Douglas-Plateau problem 873

$\partial S_{2}=L_{2}$. Let $\mathcal{S}$ be the set of rectifiable annulus $S$ such that $\partial S=L_{1} \cup L_{2}$. Douglas [fanghwang-dog1] proved that if

$$
\inf _{S \in \mathcal{S}}\{\operatorname{Area}(S)\}<\operatorname{Area}\left(S_{1}\right)+\operatorname{Area}\left(S_{2}\right),
$$

then there is an area minimizing (therefore minimal) annulus $A$ such that $\partial A=L_{1} \cup L_{2}$.

If $L_{1}$ and $L_{2}$ are coaxial unit circles in parallel planes, then it is wellknown that there is a constant $h>0$ such that when the distance between the centres is smaller than $h$, there are exactly two catenoids bounded by $L_{1} \cup L_{2}$; when the distance between the centres is equal to $h$, there is only one catenoid bounded by $L_{1} \cup L_{2}$; when the distance between the centres is larger than $h$, there are no catenoids bounded by $L_{1} \cup L_{2}$. Furthermore, by Shiffman's third theorem [fanghwang-sh1], any minimal annuli bounded by $L_{1} \cup L_{2}$ must be a rotation surface hence is a piece of a catenoid. Thus there are either two, one, or zero minimal annuli bounded by $L_{1} \cup L_{2}$ depending on the distance between their centres.

Meeks and White [fanghwang-mw1] generalized the above observation to minimal annuli bounded by two smooth convex Jordan curves $L_{1} \cup L_{2}$ in different parallel planes, i.e., there are either two, one, or zero minimal annuli bounded by $L_{1} \cup L_{2}$. But unlike the coaxial circles case, there are no simple criteria to tell us when do we have two, one, or zero minimal annuli bounded by $L_{1} \cup L_{2}$.

However, there are some partial conditions, either sufficient or necessary to the existence of a solution to some special Douglas-Plateau problems for two contours. For example, let us consider the Douglas-Plateau problem to the boundary consisting of two Jordan curves $L_{1} \cup L_{2}$ in parallel and different planes, say $L_{1} \subset P_{0}, L_{2} \subset P_{d}, d>0$.

If $L_{1}$ and $L_{2}$ are smooth convex, then besides Douglas's sufficient condition, Hoffman and Meeks in [fanghwang-hm9] gave a sufficient condition to ensure that there are two solutions, i.e., if there is a connected compact nonplanar minimal surface (could be branched) whose boundary is contained in open planar disks bounded by $L_{1}$ and $L_{2}$, then there are two minimal annuli bounded by $L_{1} \cup L_{2}$. For the precise statement, please see Theorem 2.2 below.

The result of Hoffman and Meeks can be also treated as a necessary condition, i.e., let $C_{1}$ and $C_{2}$ be smooth convex Jordan curves such that $L_{1}$ and $L_{2}$ are contained in the open planar disks bounded by $C_{1}$ and $C_{2}$ respectively, then there is a connected compact minimal surface (maybe branched) bounded by $L_{1} \cup L_{2}$ only if there are two minimal annuli bounded 
by $C_{1} \cup C_{2}$.

There are other necessary conditions. For example, Theorem 2.2 combined with a J. C. C. Nitsche's result [fanghwang-ni2], page 88, implies that the images of perpendicular projection of $L_{1}$ and $L_{2}$ on the $x y$-plane must intersect if $L_{1}$ and $L_{2}$ are convex.

Moreover, Osserman and Schiffer proved in [fanghwang-os1] that if $c, d$, $\delta_{1}$, and $\delta_{2}$ are positive constants and

$$
\begin{aligned}
& L_{1} \subset\left\{(x, y, z) ;\left(x-\frac{c}{d} z\right)^{2}+y^{2} \leq \delta_{1}^{2}, z \leq 0\right\}, \\
& L_{2} \subset\left\{(x, y, z) ;\left(x-\frac{c}{d} z\right)^{2}+y^{2} \leq \delta_{2}^{2}, z \geq d\right\},
\end{aligned}
$$

are closed curves and they bound a minimal annulus, then

$$
\delta_{1}+\delta_{2}>\sqrt{\frac{c^{2}}{2}+d^{2}} .
$$

Using the basic estimates in Lemma 2.1, in Corollary 2.2 we prove that if $\Sigma$ is a connected compact non-planar minimal surface (maybe branched) such that $\partial \Sigma=L_{1} \cup L_{2}$ and

$$
\begin{aligned}
& L_{1} \subset\left\{(x, y, z) ;-\delta_{1} \leq x-\frac{c}{d} z \leq \delta_{1}, z \leq 0\right\}, \\
& L_{2} \subset\left\{(x, y, z) ;-\delta_{2} \leq x-\frac{c}{d} z \leq \delta_{2}, z \geq d\right\},
\end{aligned}
$$

then

$$
2 \max \left\{\delta_{1}, \delta_{2}\right\}>\sqrt{c^{2}+d^{2}} .
$$

Furthermore, if

$$
\begin{aligned}
& L_{1} \subset\left\{(x, y, 0) ;-\delta_{1} \leq x \leq \delta_{1}\right\}, \\
& L_{2} \subset\left\{(x, y, d) ;-\delta_{2} \leq x-c \leq \delta_{2}\right\}
\end{aligned}
$$

then

$$
\delta_{1}+\delta_{2}>\sqrt{c^{2}+d^{2}} .
$$

We define Non-compact Douglas-Plateau problem of annular type for $n$ boundary curves as follows:

Let $L_{i}, i=1, \cdots, n$, be disjoint, embedded proper complete curves, at least one of them is non-compact, find a minimal annulus $A$ such that $\partial A=$ $\Gamma:=\bigcup_{i=1}^{n} L_{i}$. 
Curvature estimates annuli and non-compact Douglas-Plateau problem 875

As we have seen, there are many necessary conditions restricting the solvability of even compact Douglas-Plateau problems for two contours, the solvability of the non-compact Douglas-Plateau problem seems should require more hypotheses than the compact case. We will see that in fact in our special cases discussed in Section 3, the same condition that ensures the existence of solutions for compact cases is also enough for non-compact cases.

It is known for more than one hundred years that for some non-compact boundaries we can find minimal annuli solving the corresponding "two contour" Douglas-Plateau problem. A classical example is a minimal annulus bounded by two parallel straight lines, a piece of one of Riemann's examples. Although a straight line is no longer a Jordan curve, it is a proper complete (convex) curve in $\mathbb{R}^{3}$.

In [fanghwang-f3], it was proved that if $L_{1}$ and $L_{2}$ are proper noncompact complete smooth planar convex curves in parallel planes with two symmetries, then there are two minimal annuli $\mathcal{A}$ and $\mathcal{B}$ such that $\partial \mathcal{A}=\partial \mathcal{B}=\Gamma$. Furthermore, $\mathcal{A}$ and $\mathcal{B}$ are foliated by strictly convex Jordan curves.

In Section 3, we prove the existence of various types of non-compact Douglas-Plateau problems. We will show that the symmetric conditions in [fanghwang-f3] is redundant, see Theorem 3.1. The proof of Theorem 3.1 is an application of Theorem 2.1, but we must first prove that there are barriers confining the approximate compact minimal annuli such that we can use Theorem 2.1, these barriers are established in Lemma 3.1.

In Theorem 3.2 we prove that there are at least two minimal annuli bounded by four straight lines $L_{i}, i=1,2,3,4$, such that $L_{1} \subset P_{-1}$ and $L_{2} \subset P_{-1}$ are parallel, $L_{3} \subset P_{1}$ and $L_{4} \subset P_{1}$ are parallel, but $L_{1}$ and $L_{3}$ are not parallel, if the distances between $L_{1}$ and $L_{2}$, and $L_{3}$ and $L_{4}$, are sufficiently large.

We will also prove that there are minimal annuli bounded by four parallel straight lines in two different parallel planes, if the boundary satisfies some kind of Hoffman-Meeks condition. See Theorem 3.3.

Acknowledgement. We sincerely thank our colleagues Chun-Chung Hsieh, Fei-Tsen Liang, and Derchyi Wu for helpful discussions. The first author also thanks the financial support of Australian Research Council and the hospitality of the Institute of Mathematics, Academia Sinica, Taipei, this research was started and finished there during visitings. 


\section{Curvature Estimates for Minimal Annuli.}

Lemma 2.1. Let $A \subset S\left(t_{1}, t_{2}\right)$ be a minimal annulus such that $\partial A=L_{1} \cup$ $L_{2}$, where $L_{1} \subset P_{t_{1}}, L_{2} \subset P_{t_{2}}$ are convex Jordan curves. Let $\pi$ be a plane perpendicular to the $x y$-plane, $\mathcal{P}_{\pi}$ the perpendicular projection on $\pi$. Then $\Omega_{\pi}:=\operatorname{Int}\left(\mathcal{P}_{\pi}(A)\right)=\mathcal{P}_{\pi}(A)-\left(\cup_{i=1}^{4} \Gamma_{i}^{\pi}\right)$ is a domain in $\pi$ bounded by $\Gamma_{i}^{\pi}=$ $\mathcal{P}_{\pi}\left(L_{i}\right), i=1,2$, and $\Gamma_{3}^{\pi}$ and $\Gamma_{4}^{\pi}$, two curves connecting $\Gamma_{1}^{\pi}$ and $\Gamma_{2}^{\pi}$.

Let $\left|\Gamma_{i}^{\pi}\right|$ be the arc length of $\Gamma_{i}^{\pi}, i=1,2,3,4$. Then

$$
\left|\Gamma_{1}^{\pi}\right|\left|\Gamma_{2}^{\pi}\right|>\left(t_{2}-t_{1}\right)^{2}, \quad\left|\Gamma_{1}^{\pi}\right|+\left|\Gamma_{2}^{\pi}\right|>2\left(t_{2}-t_{1}\right) .
$$

In particular, if $A \subset S\left(t_{1}, t_{2}\right) \cap S^{\prime}\left(t_{1}^{\prime}, t_{2}^{\prime}\right)$, take $\pi$ to be the $y z$-plane, then from $\left|\Gamma_{i}^{\prime \pi}\right| \leq t_{2}^{\prime}-t_{1}^{\prime}, i=1$, 2, we have

$$
\min \left\{\left|\Gamma_{1}^{\pi}\right|,\left|\Gamma_{2}^{\pi}\right|\right\}>\frac{\left(t_{2}-t_{1}\right)^{2}}{\left(t_{2}^{\prime}-t_{1}^{\prime}\right)^{2}} \max \left\{\left|\Gamma_{1}^{\pi}\right|,\left|\Gamma_{2}^{\pi}\right|\right\}
$$

and

$$
\min \left\{\left|\Gamma_{1}^{\pi}\right|,\left|\Gamma_{2}^{\pi}\right|\right\}>\frac{\left(t_{2}-t_{1}\right)^{2}}{t_{2}^{\prime}-t_{1}^{\prime}}
$$

Furthermore, if $A \subset S\left(t_{1}, t_{2}\right) \cap C_{R}$, then

$$
\min \left\{\left|\Gamma_{1}^{\pi}\right|,\left|\Gamma_{2}^{\pi}\right|\right\}>\frac{\left(t_{2}-t_{1}\right)^{2}}{4 R^{2}} \max \left\{\left|\Gamma_{1}^{\pi}\right|,\left|\Gamma_{2}^{\pi}\right|\right\}
$$

and

$$
\min \left\{\left|\Gamma_{1}^{\pi}\right|,\left|\Gamma_{2}^{\pi}\right|\right\}>\frac{\left(t_{2}-t_{1}\right)^{2}}{2 R}
$$

Proof. Select a coordinate system such that $\pi$ is the $y z$-plane and

$$
\mathcal{P}_{\pi}(x, y, z)=(y, z) .
$$

For simplicity, write $\Omega_{\pi}$ as $\Omega, \mathcal{P}_{\pi}$ as $\mathcal{P}$, etc.

By Shiffman's first theorem in [fanghwang-sh1], every level curve $A \cap P_{t}$, $t_{1}<t<t_{2}$, is a strictly convex Jordan curve. Thus $\mathcal{P}\left(A \cap P_{t}\right)$ is a line 
segment and $A \cap P_{t}$ consists of two graphs on $\mathcal{P}\left(A \cap P_{t}\right), u^{+}(y, z) \geq u^{-}(y, z)$ such that $u^{+}(y, z)=u^{-}(y, z)$ if and only if $(y, z)$ is one of the two ends of $\mathcal{P}\left(A \cap P_{z}\right)$.

We orient the $y z$-plane such that $((0,1,0),(0,0,1))$ has positive orientation. Then $\Omega$ is bounded by $\Gamma_{1}=\mathcal{P}\left(A \cap P_{t_{1}}\right), \Gamma_{2}=\mathcal{P}\left(A \cap P_{t_{2}}\right)$, and $\Gamma_{3}$ and $\Gamma_{4}$ consisting of the set of end points of $\mathcal{P}\left(A \cap P_{t}\right)$ such that for any $t$, if $\left(y_{1}, t\right) \in \Gamma_{3},\left(y_{2}, t\right) \in \Gamma_{4}$, then $y_{1}<y_{2}$, thus we may say that $\Gamma_{3}$ is the left side boundary, $\Gamma_{4}$ is the right side boundary.

Recall that $\pi$ is the $y z$-plane. Let $S^{2}$ be the unit sphere in $\mathbb{R}^{3}$ and $S_{1}^{1}:=S^{2} \cap \pi$. Let $N: \operatorname{Int}(A) \rightarrow S^{2}$ be the Gauss map and $p$ be any interior point of $A$. Since $\mathcal{P}(p) \in \Gamma_{3} \cup \Gamma_{4}$ if and only if the tangent vector of $A \cap P_{t}$ at $p$ is in the direction $\pm(1,0,0)$ and since each $A \cap P_{t}$ is strictly convex, we see that $\mathcal{P}(p) \in \Gamma_{3} \cup \Gamma_{4}$ if and only if $p \in N^{-1}\left(S_{1}^{1}\right)$. Since it is proved in [fanghwang-mw1] that $N$ is one-to-one and harmonic, we know that $N^{-1}\left(S_{1}^{1}\right)$ is smooth and its tangent directions are not pointed at $\pm(1,0,0)$, therefore $\Gamma_{3} \cup \Gamma_{4}=\mathcal{P}\left(N^{-1}\left(S_{1}^{1}\right)\right)$ is smooth in its interior.

Note that for an interior point $\mathcal{P}(p)$ of $\Gamma_{3}$, the tangent line of $\Gamma_{3}$ at $\mathcal{P}(p) \in \Gamma_{3}$ is $\mathcal{P}\left(T_{p} A\right)$, where $T_{p} A$ is the tangent plane of $A$ at $p$. Since $A$ is minimal, there are points of $A$ at both sides of $T_{p} A$ in any neighbourhood of $p$ in $\mathbb{R}^{3}$. Thus since $\Gamma_{3}$ is the left side boundary of $\Omega$, there are points of $\bar{\Omega}$ on the left side of $\mathcal{P}\left(T_{p} A\right)$ in any neighbourhood of $\mathcal{P}(p)$, hence $\Gamma_{3}$ is locally on the left side of its tangent line at $\mathcal{P}(p)$. If $\Gamma_{3}$ is not convex, then there is another point $\mathcal{P}\left(p_{1}\right) \in \Gamma_{3} \cap \mathcal{P}\left(T_{p} A\right)$. Thus there would be another point $\mathcal{P}(q)$ in the interior of $\Gamma_{3}$ and is located between $\mathcal{P}(p)$ and $\mathcal{P}\left(p_{1}\right)$ such that $\mathcal{P}(q)$ is on the left side of $\mathcal{P}\left(T_{p} A\right)$ and the distance from $\mathcal{P}(q)$ to $\mathcal{P}\left(T_{p} A\right)$ is a local maximum, thus $T_{q} A$ is parallel to $T_{p} A$. Therefore in a small neighbourhood of $q$ in $\mathbb{R}^{3}$ there are no points of $A$ on the left side of $T_{q}(A)$, a contradiction. Similarly we can prove that $\Gamma_{4}$ is convex.

We now prove that $\mathcal{P}(A)-\bigcup_{i=1}^{4} \Gamma_{i}$ is a domain and $\Omega=\mathcal{P}(A)-\bigcup_{i=1}^{4} \Gamma_{i}$. In fact if $\mathcal{P}(p) \notin \Gamma_{1} \cup \Gamma_{2} \cup \Gamma_{3} \cup \Gamma_{4}$, then $p \notin N^{-1}\left(S_{1}^{1}\right)$, there is a neighbourhood $U$ of $p$ in $\mathbb{R}^{3}$ such that $U \cap N^{-1}\left(S_{1}^{1}\right)=\emptyset$ and $\mathcal{P}(U \cap A)$ is open in $\pi$ and $\mathcal{P}(U \cap A) \cap\left(\Gamma_{1} \cup \Gamma_{2} \cup \Gamma_{3} \cup \Gamma_{4}\right)=\emptyset$, i.e., $\mathcal{P}(p) \in \operatorname{Int}(\mathcal{P}(A))$, hence $\Omega=$ $\operatorname{Int}(\mathcal{P}(A))=\mathcal{P}(A)-\left(\bigcup_{i=1}^{4} \Gamma_{i}\right)$ and $\partial \Omega=\bigcup_{i=1}^{4} \Gamma_{i}$. Finally, $\Omega$ is connected since $\mathcal{P}(A)$ is connected and $\bigcup_{i=1}^{4} \Gamma_{i}$ does not separate $\mathcal{P}(A)$. See Figure 1 below. 

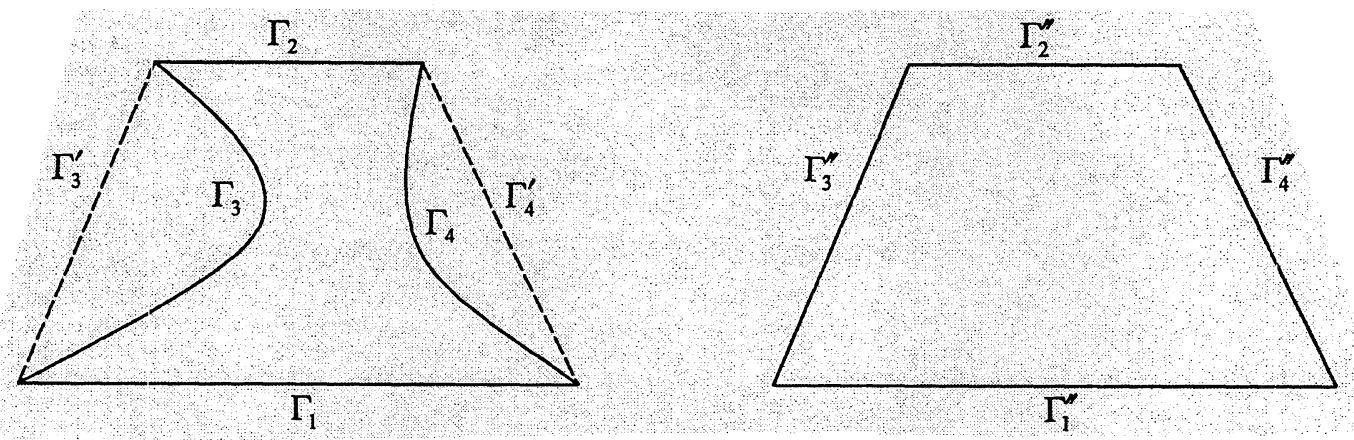

Figure 1

Since $\Gamma_{1}$ and $\Gamma_{2}$ are straight line segments, $\Omega$ is a domain with piecewise smooth boundary and $\partial \Omega$ has only four corner points $\left[\Gamma_{1} \cap\left(\Gamma_{3} \cup \Gamma_{4}\right)\right] \cup\left[\Gamma_{2} \cap\right.$ $\left.\left(\Gamma_{3} \cup \Gamma_{4}\right)\right]$. Let $\nu$ be the outward unit normal vector of $\partial \Omega$.

Let us consider the graph defined by $u=u^{+}$. Then $u$ satisfies the minimal surface equation $\operatorname{div} T u=0$, where $T u=D u / \sqrt{1+|D u|^{2}}$. The Gauss map is given by

$$
N(x, y, z)=\frac{1}{\sqrt{1+|D u|^{2}}}(1,-D u)(y, z), \quad(y, z) \in \Omega, \quad x=u(y, z) .
$$

If $\mathcal{P}(p)$ is an interior point of $\Gamma_{3} \cup \Gamma_{4}$, then since $N(p)=(0, b, c), \mathcal{P}(N(p))=$ $N(p)$. Since $N(p)$ is perpendicular to the tangent vector $v(p)$ along $N^{-1}\left(S_{1}^{1}\right)$ at $p, N(p)$ is also perpendicular to the tangent vector $\tilde{v}((\mathcal{P}(p))=\mathcal{P}(v(p))$ along $\Gamma_{3}$ or $\Gamma_{4}$ at $\mathcal{P}(p)$, thus $\nu(\mathcal{P}(p))=\mathcal{P}(N(p))$. We then have that

$$
\nu(\mathcal{P}(p))=\mathcal{P}(N(p))=N(p)=(0, b, c) .
$$

Therefore, $\nu \bullet N=1$ along the interior of $\Gamma_{3}$ and $\Gamma_{4}$. Thus by the expression of $N$ we see that $u$ satisfies the boundary condition

$$
\nu \bullet T u=-\nu \bullet N=-1, \quad \text { on } \quad \Gamma_{3} \cup \Gamma_{4} .
$$

First assume that $L_{1}$ and $L_{2}$ are smooth and strictly convex, then $D u$ exists on $\Gamma_{1} \cup \Gamma_{2}$. We have

$$
\int_{\partial \Omega} T u \bullet \nu=\int_{\Omega} \operatorname{div} T u=0
$$


Curvature estimates annuli and non-compact Douglas-Plateau problem 879

Now since $|T u \bullet \nu| \leq 1$,

$$
\left|\Gamma_{3}\right|+\left|\Gamma_{4}\right|=-\int_{\Gamma_{3} \cup \Gamma_{4}} T u \bullet \nu=\int_{\Gamma_{1} \cup \Gamma_{2}} T u \bullet \nu \leq\left|\Gamma_{1}\right|+\left|\Gamma_{2}\right| .
$$

If $L_{1}$ or $L_{2}$ is only continuously convex, then by Shiffman's theorem $A \cap P_{t}$ is smooth and strictly convex for any $t \in\left(t_{1}, t_{2}\right)$. Consider $A \cap S\left(t_{1}+\epsilon, t_{2}-\epsilon\right)$, $0<\epsilon<\left(t_{2}-t_{1}\right) / 2$, then (2.1) is true to the corresponding $\Gamma_{i}$ 's of $A \cap S\left(t_{1}-\right.$ $\left.\epsilon, t_{2}+\epsilon\right)$. Since $A$ is continuous up to boundary, letting $\epsilon \rightarrow 0$, we have proved (2.1).

To prove (2.2) we replace $\Gamma_{3}$ by the line segment $\Gamma_{3}^{\prime}$ connecting the two end points of $\Gamma_{3}$, replace $\Gamma_{4}$ by the line segment $\Gamma_{4}^{\prime}$ connecting the two end points of $\Gamma_{4}$. Then by comparison principle for minimal surfaces, $\left|\Gamma_{3}^{\prime}\right|<\left|\Gamma_{3}\right|$, $\left|\Gamma_{4}^{\prime}\right|<\left|\Gamma_{4}\right|$. Furthermore we replace the four-gon $\Gamma_{1} \cup \Gamma_{2} \cup \Gamma_{3}^{\prime} \cup \Gamma_{4}^{\prime}$ by a trapezoid $\Gamma_{1}^{\prime \prime} \cup \Gamma_{2}^{\prime \prime} \cup \Gamma_{3}^{\prime \prime} \cup \Gamma_{4}^{\prime \prime}$ such that $\left|\Gamma_{3}^{\prime \prime}\right|=\left|\Gamma_{4}^{\prime \prime}\right|$, and $\left|\Gamma_{1}^{\prime \prime}\right|=\left|\Gamma_{1}\right|,\left|\Gamma_{2}^{\prime \prime}\right|=\left|\Gamma_{2}\right|$ and $\Gamma_{1}^{\prime \prime}$ and $\Gamma_{2}^{\prime \prime}$ are parallel.

Note that $\left|\Gamma_{3}^{\prime \prime}\right|=\left|\Gamma_{4}^{\prime \prime}\right|$ and $2\left|\Gamma_{3}^{\prime \prime}\right| \leq\left|\Gamma_{3}^{\prime}\right|+\left|\Gamma_{4}^{\prime}\right|<\left|\Gamma_{3}\right|+\left|\Gamma_{4}\right| \leq\left|\Gamma_{1}\right|+\left|\Gamma_{2}\right|$. Now let $h=t_{2}-t_{1}$ and without loss of generality suppose that $\left|\Gamma_{1}\right| \geq\left|\Gamma_{2}\right|$, thus $\left|\Gamma_{2}\right|=c\left|\Gamma_{1}\right|, 0<c \leq 1$, and $2\left|\Gamma_{3}^{\prime \prime}\right|<(1+c)\left|\Gamma_{1}\right|$. Since

$$
\left|\Gamma_{3}^{\prime \prime}\right|=\sqrt{\frac{\left(\left|\Gamma_{1}\right|-\left|\Gamma_{2}\right|\right)^{2}}{4}+h^{2}}=\sqrt{\frac{(1-c)^{2}\left|\Gamma_{1}\right|^{2}}{4}+h^{2}},
$$

we obtain that

$$
(1-c)^{2}\left|\Gamma_{1}\right|^{2}+4 h^{2}<(1+c)^{2}\left|\Gamma_{1}\right|^{2} .
$$

Thus

$$
h^{2}<c\left|\Gamma_{1}\right|^{2}=\left|\Gamma_{1}\right|\left|\Gamma_{2}\right| .
$$

By comparison principle for minimal surfaces,

$$
2 h \leq\left|\Gamma_{3}^{\prime}\right|+\left|\Gamma_{4}^{\prime}\right|<\left|\Gamma_{3}\right|+\left|\Gamma_{4}\right| \leq\left|\Gamma_{1}\right|+\left|\Gamma_{2}\right|,
$$

(2.2) is proved.

If $A \subset S\left(t_{1}, t_{2}\right) \cap S^{\prime}\left(t_{1}^{\prime}, t_{2}^{\prime}\right)$, then $\left|\Gamma_{1}\right| \leq t_{2}^{\prime}-t_{1}^{\prime}$, so by $(2.7)$

$$
c>\frac{h^{2}}{\left(t_{2}^{\prime}-t_{1}^{\prime}\right)^{2}}
$$

(2.3) is true. From (2.2),

$$
\left|\Gamma_{2}\right|>\frac{h^{2}}{\left|\Gamma_{1}\right|} \geq \frac{h^{2}}{t_{2}^{\prime}-t_{1}^{\prime}}
$$


(2.4) is true.

If $A \subset S\left(t_{1}, t_{2}\right) \cap C_{R}$, then $t_{2}^{\prime}-t_{1}^{\prime} \leq 2 R$. By a rotation, we see that (2.5) and (2.6) are true.

The proof is now complete.

Similar arguments as in Lemma 2.1 give us further information of the domain $\operatorname{Int}(\mathcal{P}(A))$.

Lemma 2.2. Suppose $A \subset S\left(t_{1}, t_{2}\right) \cap S^{\prime}(-R, R)$ is a minimal annulus such that $\partial A=L_{1} \cup L_{2}$, where $L_{1} \subset P_{t_{1}}$ and $L_{2} \subset P_{t_{2}}$ are convex Jordan curves. Let $\pi$ be the yz-plane and $\mathcal{P}_{\pi}$ the perpendicular projection on $\pi$. Let $\Omega^{\pi}=$ $\operatorname{Int}\left(\mathcal{P}_{\pi}(A)\right)$ be the domain in $\pi$ and $\partial \Omega^{\pi}=\Gamma_{1}^{\pi} \cup \Gamma_{2}^{\pi} \cup \Gamma_{3}^{\pi} \cup \Gamma_{4}^{\pi}$ be as in Lemma 2.1. Let $p$ be any interior point of $A$ and $d=\operatorname{dist}\left(p, \partial S\left(t_{1}, t_{2}\right)\right)$. Then

$$
d^{\prime}:=\max \left\{\operatorname{dist}\left(\mathcal{P}_{\pi}(p), \Gamma_{3}^{\pi}\right), \operatorname{dist}\left(\mathcal{P}_{\pi}(p), \Gamma_{4}^{\pi}\right)\right\}>\frac{(\sqrt{2}-1) d^{2}}{R} .
$$

Proof. Let us write $\Omega^{\pi}=\Omega$ etc.. Since $\Gamma_{3}$ and $\Gamma_{4}$ are compact, there are $q_{3} \in \Gamma_{3}$ and $q_{4} \in \Gamma_{4}$ such that $\left|\mathcal{P}(p)-q_{3}\right|=\operatorname{dist}\left(\mathcal{P}(p), \Gamma_{3}\right),\left|\mathcal{P}(p)-q_{4}\right|=$ $\operatorname{dist}\left(\mathcal{P}(p), \Gamma_{4}\right)$. Connecting $\mathcal{P}(p)$ and $q_{3}, \mathcal{P}(p)$ and $q_{4}$ by line segments $l_{3}$ and $l_{4}$ to form two subdomains $\Omega_{1}$ and $\Omega_{2}$ such that $\Omega=\Omega_{1} \cup \Omega_{2} \cup l_{3} \cup l_{4}$ and $\partial \Omega_{1}=\Gamma_{1} \cup l_{3} \cup l_{4} \cup \Gamma_{3}^{\prime} \cup \Gamma_{4}^{\prime}, \partial \Omega_{2}=\Gamma_{2} \cup l_{3} \cup l_{4} \cup \Gamma_{3}^{\prime \prime} \cup \Gamma_{4}^{\prime \prime}$, where $\Gamma_{i}^{\prime} \subset \Gamma_{i}$, $\Gamma_{i}^{\prime \prime} \subset \Gamma_{i}$, for $i=3$, 4. Note that $l_{3}$ and $l_{4}$ are contained in $\Omega$ except at end points on $\Gamma_{3}$ and $\Gamma_{4}$ so that $\Gamma_{i}^{\prime} \cup \Gamma_{i}^{\prime \prime}=\Gamma_{i}$, and $\left|\Gamma_{i}^{\prime}\right|+\left|\Gamma_{i}^{\prime \prime}\right|=\left|\Gamma_{i}\right|$, for $i=3$, 4. See Figure 2 below.

Let $p=(x, y, z), t_{1}<z<t_{2}$. Let $T_{1}$ and $T_{2}$ be the line segments connecting $\mathcal{P}(p)$ and the two ends of $\Gamma_{1}$, then

$$
\left|T_{1}\right|+\left|T_{2}\right|<2 d^{\prime}+\left|\Gamma_{3}^{\prime}\right|+\left|\Gamma_{4}^{\prime}\right| \text {. }
$$

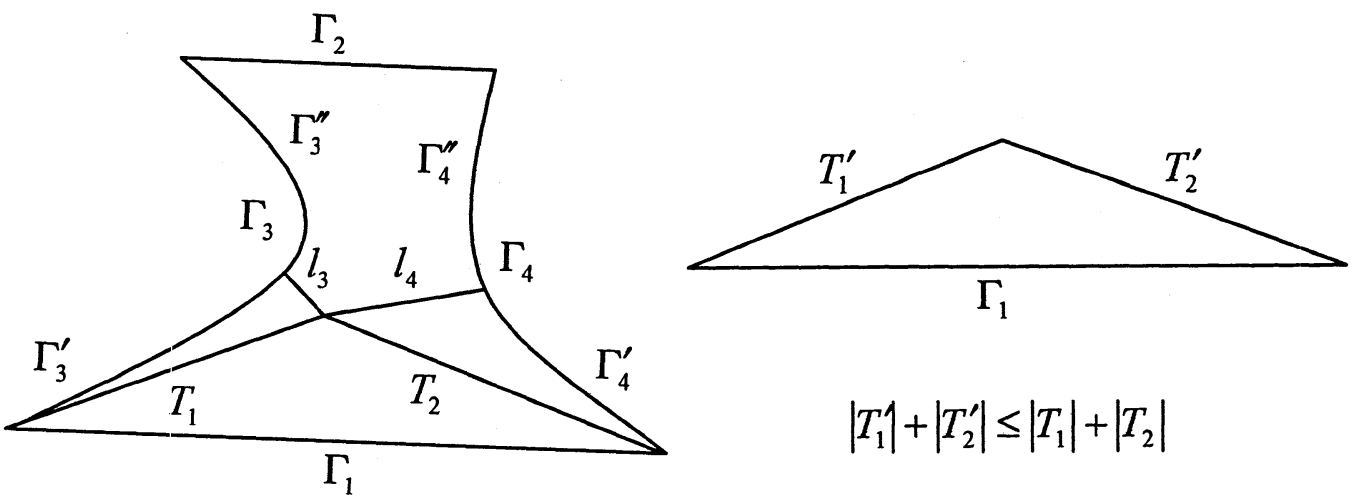

Figure 2 
Curvature estimates annuli and non-compact Douglas-Plateau problem 881

Elementary geometry tells us that

$$
\left|T_{1}\right|+\left|T_{2}\right| \geq 2 \sqrt{\frac{\left|\Gamma_{1}\right|^{2}}{4}+\left(z-t_{1}\right)^{2}},
$$

thus

$$
2 d^{\prime}+\left|\Gamma_{3}^{\prime}\right|+\left|\Gamma_{4}^{\prime}\right|>2 \sqrt{\frac{\left|\Gamma_{1}\right|^{2}}{4}+\left(z-t_{1}\right)^{2}}=\left|\Gamma_{1}\right| \sqrt{1+\frac{4\left(z-t_{1}\right)^{2}}{\left|\Gamma_{1}\right|^{2}}}
$$

Similarly, we have

$$
2 d^{\prime}+\left|\Gamma_{3}^{\prime \prime}\right|+\left|\Gamma_{4}^{\prime \prime}\right|>2 \sqrt{\frac{\left|\Gamma_{2}\right|^{2}}{4}+\left(t_{2}-z\right)^{2}}=\left|\Gamma_{2}\right| \sqrt{1+\frac{4\left(t_{2}-z\right)^{2}}{\left|\Gamma_{2}\right|^{2}}} .
$$

Now since $d=\min \left\{\left(z-t_{1}\right),\left(t_{2}-z\right)\right\} \leq\left(t_{2}-t_{1}\right) / 2$ and $\max \left\{\left|\Gamma_{1}\right|,\left|\Gamma_{2}\right|\right\} \leq 2 R$, by (2.2) and (2.6),

$$
\min \left\{\left|\Gamma_{1}\right| R,\left|\Gamma_{2}\right| R\right\} \geq \frac{\left|\Gamma_{1}\right|\left|\Gamma_{2}\right|}{2} \geq \frac{\left(t_{2}-t_{1}\right)^{2}}{2} \geq 2 d^{2},
$$

thus

$$
\max \left\{\frac{2 d^{2}}{\left|\Gamma_{1}\right| R}, \frac{2 d^{2}}{\left|\Gamma_{2}\right| R}\right\} \leq 1 .
$$

Note that $\sqrt{1+x} \geq 1+(\sqrt{2}-1) x$ for $0 \leq x \leq 1$, we obtain

$$
\begin{gathered}
\left|\Gamma_{1}\right| \sqrt{1+\frac{4\left(z-t_{1}\right)^{2}}{\left|\Gamma_{1}\right|^{2}}} \geq\left|\Gamma_{1}\right| \sqrt{1+\frac{2 d^{2}}{\left|\Gamma_{1}\right| R}} \geq\left|\Gamma_{1}\right|\left(1+\frac{2(\sqrt{2}-1) d^{2}}{\left|\Gamma_{1}\right| R}\right) \\
=\left|\Gamma_{1}\right|+\frac{2(\sqrt{2}-1) d^{2}}{R} \\
\left|\Gamma_{2}\right| \sqrt{1+\frac{4\left(t_{2}-z\right)^{2}}{\left|\Gamma_{2}\right|^{2}}} \geq\left|\Gamma_{2}\right|+\frac{2(\sqrt{2}-1) d^{2}}{R} .
\end{gathered}
$$

Therefore,

$$
\begin{aligned}
4 d^{\prime}+\left|\Gamma_{3}\right|+\left|\Gamma_{4}\right| & >\left|\Gamma_{1}\right| \sqrt{1+\frac{4\left(z-t_{1}\right)^{2}}{\left|\Gamma_{1}\right|^{2}}}+\left|\Gamma_{2}\right| \sqrt{1+\frac{4\left(t_{2}-z\right)^{2}}{\left|\Gamma_{2}\right|^{2}}} \\
& \geq\left|\Gamma_{1}\right|+\left|\Gamma_{2}\right|+\frac{4(\sqrt{2}-1) d^{2}}{R}
\end{aligned}
$$


But by (2.1)

$$
\left|\Gamma_{1}\right|+\left|\Gamma_{2}\right| \geq\left|\Gamma_{3}\right|+\left|\Gamma_{4}\right|
$$

we obtain that

$$
d^{\prime}>\frac{(\sqrt{2}-1) d^{2}}{R}
$$

From Lemma 2.2 we obtain an interior estimate of curvature.

Proposition 2.1 (Interior Curvature Estimate). If $A \subset S\left(t_{1}, t_{2}\right) \cap C_{R}$ is a minimal annulus such that $\partial A=L_{1} \cup L_{2}$, where $L_{1} \subset P_{t_{1}}$ and $L_{2} \subset P_{t_{2}}$ are convex Jordan curves, then there is an absolute constant $C_{0}>0$, such that

$$
|K(p)| \leq \frac{C_{0} R^{2}}{d^{4}}
$$

where $d^{\prime}=\operatorname{dist}\left(p, \partial S\left(t_{1}, t_{2}\right)\right)$.

Proof. Let $\mathcal{P}_{\theta}$ be the projection on a plane $\pi_{\theta}$ with normal

$$
v_{\theta}=(\cos \theta, \sin \theta, 0) .
$$

Then by Lemma $2.1 \operatorname{Int}\left(\mathcal{P}_{\theta}(A)\right)$ is a domain bounded by $\Gamma_{i}^{\theta}, i=1,2,3$, 4. We give an orthonormal basis in $\pi_{\theta},\left(e_{1}, e_{2}\right)$, such that $\left(v_{\theta}, e_{1}, e_{2}\right)$ is an positive basis of $\mathbb{R}^{3}$. The convention is that under this coordinate system, $\Gamma_{3}^{\theta}$ has a smaller first coordinate than that for $\Gamma_{4}^{\theta}$. Define

$$
f(\theta)=\operatorname{dist}\left(\mathcal{P}_{\theta}(p), \Gamma_{3}^{\theta}\right)-\operatorname{dist}\left(\mathcal{P}_{\theta}(p), \Gamma_{4}^{\theta}\right) .
$$

Then $f$ is continuous and $f(\theta+\pi)=-f(\theta)$. Thus there exists at least one $\theta=\theta_{0}$, such that $f\left(\theta_{0}\right)=0$, i.e., $\operatorname{dist}\left(\mathcal{P}_{\theta_{0}}(p), \Gamma_{3}^{\theta_{0}}\right)=\operatorname{dist}\left(\mathcal{P}_{\theta_{0}}(p), \Gamma_{4}^{\theta_{0}}\right)=d^{\prime}$.

By Lemma $2.2, d^{\prime}>D:=(\sqrt{2}-1) d^{2} / R$, where $d=\operatorname{dist}\left(p, \partial S\left(t_{1}, t_{2}\right)\right)$. Thus there is a round disk centred at $\mathcal{P}(p)$ of radius greater than or equal to $r=\min \{D, d\}$ contained in $\Omega$ and $p=(u(\mathcal{P}(p)), \mathcal{P}(p))$ is on a minimal graph generated by $u=u^{+}$or $u^{-}$. A theorem of Osserman [fanghwang-os1], page 107, says that there is an absolute constant $C>0$ such that

$$
|K(p)| \leq \frac{C}{r^{2}}
$$


Curvature estimates annuli and non-compact Douglas-Plateau problem 883

Finally by (2.2) and the definition of $d$,

$$
4 R \geq\left|\Gamma_{1}^{\theta_{0}}\right|+\left|\Gamma_{2}^{\theta_{0}}\right|>2\left(t_{2}-t_{1}\right) \geq 4 d>4(\sqrt{2}-1) d,
$$

we know that $\min \{D, d\}=D$. Taking $C_{0}=\frac{C}{(\sqrt{2}-1)^{2}}$, we complete the proof.

Recall that the Gaussian curvature bound ensures compactness, as stated in the following summarization appeared in [fanghwang-ands],

Lemma 2.3 (Compactness Lemma). Let $\Omega$ be a bounded domain in a complete Riemannian 3-manifold $\mathbf{N}^{3}$ and let $\left\{M_{i}\right\}$ be a sequence of minimally immersed surfaces in $\Omega$. Suppose there is a constant $C$ such that the Gauss curvature $K_{M_{i}}(x)$ satisfies $\left|K_{M_{i}}(x)\right|<C$ for all $i$. Then a subsequence of $\left\{M_{i}\right\}$ converges smoothly (in the $C^{k}$-topology, $k \geq 2$ ) to an immersed minimal surface $M_{\infty}$ (with multiplicity) in $\Omega$ and $\left|K_{M_{\infty}}(x)\right| \leq C$. If each $M_{i}$ is embedded, then $M_{\infty}$ is also embedded.

Theorem 2.1. Let $\left\{A_{n}\right\}$ be a sequence of embedded minimal annuli contained in $S\left(t_{1}, t_{2}\right) \cap C_{R}$ such that $\partial A_{n}=L_{1}^{n} \cup L_{2}^{n}$, where $L_{1}^{n} \subset P_{t_{1}}, L_{2}^{n} \subset P_{t_{2}}$ are convex Jordan curves. Then there is a subsequence of $\left\{A_{n}\right\}$ converging to an embedded minimal annulus $A \subset S\left(t_{1}, t_{2}\right) \cap C_{R}$ such that $\partial A=L_{1} \cup L_{2}$, where $L_{1} \subset P_{t_{1}}, L_{2} \subset P_{t_{2}}$ are convex Jordan curves.

Proof. First observe that as uniformly bounded convex Jordan curves $\left\{L_{1}^{n}\right\}$ and $\left\{L_{2}^{n}\right\}$ are equicontinuous.

In fact, by Lemma 2.1, the arc lengths of $\left\{L_{1}^{n}\right\}$ and $\left\{L_{2}^{n}\right\}$ are at least $\left(t_{2}-t_{1}\right)^{2} / R$. Since $\left\{L_{1}^{n}\right\}$ and $\left\{L_{2}^{n}\right\}$ are contained in $C_{R}$ and are convex, their arc lengths have an upper bound too.

Thus a subsequence of $\left\{L_{1}^{n}\right\}$, still denote by $\left\{L_{1}^{n}\right\}$, has a convergent arc length, i.e., $l_{n}:=\left|L_{1}^{n}\right| \rightarrow l \geq\left(t_{2}-t_{1}\right)^{2} / R$.

Since $L_{1}^{n}$ is convex, it has tangent almost everywhere. Let $\alpha_{n}:\left[0, l_{n}\right] \rightarrow$ $P_{t_{1}}$ be the embedding of $L_{1}^{n}$ such that $\left|\dot{\alpha}_{n}\right|=1$ almost everywhere. Define $s$ : $[0, l] \rightarrow\left[0, l_{n}\right]$ by $s(t)=l_{n} t / l$ and $\beta_{n}:[0, l] \rightarrow P_{t_{1}}$ by $\beta_{n}(t)=\alpha_{n}(s(t))$, then $\left|\dot{\beta}_{n}\right|=l_{n} / l$ almost everywhere. Thus $\left\{\beta_{n}\right\}$ is equicontinuous and uniformly bounded.

By Ascoli-Arzela theorem, a subsequence of $\beta_{n}$, still denote by $\beta_{n}$, uniformly converges to a continuous mapping $\beta:[0, l]$. By (2.6) the image of $\beta$ cannot be a line segment or a point. Since each $\beta_{n}$ is one-to-one except at the two ends, $\beta$ defines a Jordan curve $L_{1}$. Let $D_{1}$ be the domain enclosed 
by $L_{1}$ and $D_{1}^{n}$ the domain enclosed by $L_{1}^{n}$, then $D_{1}^{n} \rightarrow D_{1}$. Since each $D_{1}^{n}$ is convex, $D_{1}$ is convex. Hence $L_{1}$ is a convex Jordan curve. Similarly we can treat $\left\{L_{2}^{n}\right\}$ and we may assume that a subsequence of $\left\{\partial A_{n}\right\}$, still denote by $\left\{\partial A_{n}\right\}$, has a limit $L_{1} \cup L_{2}$, and $L_{1} \subset P_{t_{1}}$ and $L_{2} \subset P_{t_{2}}$ are convex Jordan curves.

Let $\epsilon_{m} \searrow 0$ as $m \rightarrow \infty$ and $A_{n}^{m}=A_{n} \cap S\left(t_{1}+\epsilon_{m}, t_{2}-\epsilon_{m}\right) \subset C_{R}$. Then by Proposition 2.1 for fixed $m,\left\{A_{n}^{m}\right\}$ has a uniform curvature bound, thus by Lemma 2.3 , there is a subsequence $\left\{A_{m_{n}}^{m}\right\}$ converging in $S\left(t_{1}+\epsilon_{m}, t_{2}-\right.$ $\left.\epsilon_{m}\right) \cap C_{R}$. Thus the subsequence $\left\{A_{m_{m}}\right\}$ converges in the interior of $S\left(t_{1}, t_{2}\right)$ to an open minimal surface $A$.

Since each $A_{m_{m}} \cap P_{t}$ is a strictly convex Jordan curve in $C_{R} \cap P_{t}$ and $\left\{A_{m_{m}}\right\}$ converges in $C^{k}$ topology, $k \geq 2, A \cap P_{t}$ must be a convex Jordan curve, thus $A$ is a minimal annulus.

Now $\lim _{m \rightarrow \infty} \partial A_{m_{m}}=L_{1} \cup L_{2}$. We only need prove that $\partial A=L_{1} \cup L_{2}$.

In fact, let $\mathcal{P}_{\pi}$ be the perpendicular projection on a plane $\pi$ perpendicular to the $x y$-plane and $\Omega_{m}^{\pi}=\operatorname{Int}\left(\mathcal{P}_{\pi}\left(A_{m_{m}}\right)\right)$, then by Lemma $2.1, A_{m_{m}}$ consists of two simply connected graphs $G_{m}^{+}$and $G_{m}^{-}$with continuous boundary. Similarly, $A$ consists of two simply connected graphs $G^{+}$and $G^{-}$on a domain in the plane $\pi$.

Let $X_{m}^{+}: D \rightarrow \mathbb{R}^{3}$ and $X_{m}^{-}: D \rightarrow \mathbb{R}^{3}$ be conformal embeddings from the closed unit disk $D$ for $G_{m}^{+}$and $G_{m}^{-}$respectively, normalized such that $X_{m}^{+}\left(p_{i}\right)=q_{i}^{m}$, for three fixed points $p_{i} \in \partial D$ and $\lim _{m \rightarrow \infty} q_{i}^{m}=q_{i} \in L_{1}$. Similarly we require the three points condition for $X_{m}^{-}$.

Since $\partial A_{m_{m}}$ consists of two convex Jordan curves in $\partial S\left(t_{1}, t_{2}\right) \cap C_{R}$, the arc lengths of $\partial A_{m_{m}}$ is uniformly bounded, hence by isoperimetric inequality, the areas of $A_{m_{m}}$, hence of $G_{m}^{+}, G_{m}^{-}$, are uniformly bounded. Now since $X_{m}^{+}$ are conformal, $\int_{D}\left|D X_{m}^{+}\right|^{2}$ is uniformly bounded. Therefore, by CourantLebesgue Lemma (see Theorem 3 on page 238 of [fanghwang-dhkw]), $X_{m}^{+}$is uniformly continuous and converges on $D$.

Similarly we can prove that $X_{m}^{-}$converges on $D$. Since $G_{m}^{+} \rightarrow G^{+}$and $G_{m}^{-} \rightarrow G^{-}$, and $\partial\left(G_{m}^{+} \cup G_{m}^{-}\right) \cap \partial S\left(t_{1}, t_{2}\right)=\partial A_{m_{m}}$ converges to $L_{1} \cup L_{2}$, and the plane $\pi$ was arbitrary, we see that $\partial A=L_{1} \cup L_{2}$ and $\bar{A}$ is continuous up to boundary and $\partial A=L_{1} \cup L_{2}$.

Remark 2.1. The argument used in the proof that $\bar{A}$ is continuous actually gives an alternative proof that a subsequence of $\left\{A_{n}\right\}$ converges to a minimal annulus without curvature estimates and Lemma 2.3 , i.e., via the uniformly boundedness of $\int_{D}\left|D X_{m}\right|^{2}$ and the Courant-Lebesgue Lemma, with the help of Lemma 2.1 and 2.2. 
Curvature estimates annuli and non-compact Douglas-Plateau problem 885

With Theorem 2.1, we can give a generalization of a theory dealing with smooth convex boundary developed by Hoffman and Meeks in [fanghwang$\mathrm{hm} 9$ ], and Meeks and White in [fanghwang-mw1], to the continuous convex boundary case.

Theorem 2.2. Suppose $D_{1}$ and $D_{2}$ are two open disks lying on parallel planes, and suppose their boundaries $L_{1}$ and $L_{2}$ are continuous convex Jordan curves.

1. If $A^{\prime}$ is a connected non-planar compact (maybe branched) minimal surface such that $\partial A^{\prime} \subset D_{1} \cup D_{2}$, then there exist at least two embedded compact minimal annuli $A$ and $B, \partial A=\partial B=L_{1} \cup L_{2}$.

2. $A$ is stable and has the property that for any disks $D^{\prime} \subset D_{1}$ and $D^{\prime \prime} \subset$ $D_{2}$ with continuous boundaries, if there is a connected compact (maybe branched) minimal surface $N$ such that $\partial N=\partial D^{\prime} \cup \partial D^{\prime \prime}$, then $N$ is contained in the solid $V$ bounded by $A \cup D_{1} \cup D_{2}$. In particular, if $A \neq N$, then $\operatorname{Int}(A) \cap \operatorname{Int}(N)=\emptyset$. On the other hand, $B$ is unstable and $\operatorname{Int}(B) \cap \operatorname{Int}(N) \neq \emptyset$.

3. If merely $\partial A^{\prime} \subset \overline{D_{1}} \cup \overline{D_{2}}$, then there exists at least one embedded minimal annulus $C$ such that $\partial C=L_{1} \cup L_{2}$. Such a $C$ is almost stable in the sense that the first eigenvalue of the second variation of $C$ is larger than or equal to zero. Let $N$ be a connected compact (maybe branched) minimal surface such that $\partial N=\partial D^{\prime} \cup \partial D^{\prime \prime}$, then $N$ is contained in the solid $V$ bounded by $C \cup D_{1} \cup D_{2}$. In particular, if $C \neq N$, then $\operatorname{Int}(C) \cap \operatorname{Int}(N)=\emptyset$.

4. Furthermore, the symmetry groups of $A$ and $B$, or $C$, are the same as the symmetry group of $L_{1} \cup L_{2}$.

Proof. If $L_{1}$ and $L_{2}$ are smooth, or in the cases of existence of $A$ and $C$ in conclusions 1 or 3 for merely continuous $L_{1}$ and $L_{2}$, the theorem is a combination of Theorem 1.1, 1.2 of [fanghwang-hm9], and Lemma 2.1 of [fanghwang-mw1], with "exact" replacing "at least" in 1.

In general for the case of $L_{1}$ and $L_{2}$ are merely continuous, let $\operatorname{Symm}\left(L_{1} \cup\right.$ $L_{2}$ ) be the symmetry group of $L_{1} \cup L_{2}$. We can construct smooth convex Jordan curves $L_{1}^{n}$ and $L_{2}^{n}$ such that $\lim _{n \rightarrow \infty} L_{1}^{n}=L_{1}, \lim _{n \rightarrow \infty} L_{2}^{n}=L_{2}$, $\operatorname{Symm}\left(L_{1}^{n} \cup L_{2}^{n}\right)=\operatorname{Symm}\left(L_{1} \cup L_{2}\right)$, and $L_{1}$ and $L_{2}$ are enclosed in the disks bounded by $L_{1}^{n}$ and $L_{2}^{n}$ respectively. Therefore there are corresponding minimal annuli $A_{n}$ and $B_{n}$ etc., satisfying all the properties stated in 
the theorem, and being contained in some $C_{R}$. Using Theorem 2.1 we get our limiting minimal annuli $A$ and $B$ bounded by $L_{1} \cup L_{2}$, they satisfy all properties stated in the theorem, especially, $A \neq B$.

An immediate corollary of Lemma 2.1 and Theorem 2.2 is

Corollary 2.1. If $\Sigma \subset S\left(t_{1}, t_{2}\right)$ is a connected compact non-planar minimal surface (maybe branched) such that $\partial \Sigma \subset P_{t_{1}} \cup P_{t_{2}}$. Let $L(\theta, t)$ be the length of $\Sigma \cap P_{t}$ projected on the plane with normal $(\cos \theta, \sin \theta, 0)$, for $(\theta, t) \in$ $[0, \pi] \times\left[t_{1}, t_{2}\right]$. Then for any $t_{1} \leq s<t \leq t_{2}$ and $\theta \in[0, \pi]$,

$$
L(\theta, s)+L(\theta, t)>2(t-s), \quad L(\theta, s) L(\theta, t)>(t-s)^{2} .
$$

Thus

$$
L(\theta, t)>\max \left\{\frac{\left(t-t_{1}\right)^{2}}{L\left(\theta, t_{1}\right)}, \frac{\left(t_{2}-t\right)^{2}}{L\left(\theta, t_{2}\right)}\right\}, \quad t \in\left(t_{1}, t_{2}\right) .
$$

In particular, if $\partial \Sigma \subset S\left(t_{1}, t_{2}\right) \cap S^{\prime}\left(t_{1}^{\prime}, t_{2}^{\prime}\right)$, then $t_{2}^{\prime}-t_{1}^{\prime}>t_{2}-t_{1}$. Furthermore, if $\Sigma \subset S\left(t_{1}, t_{2}\right) \cap C_{R}$, then

$$
L(\theta, t)>\max \left\{\frac{\left(t-t_{1}\right)^{2}}{2 R}, \frac{\left(t_{2}-t\right)^{2}}{2 R}\right\}, \quad t \in\left(t_{1}, t_{2}\right) .
$$

Proof. First observe that by comparison principle for minimal surfaces $\Sigma \cap P_{t}$ is a variety without isolated points, hence we can apply Theorem 2.2. Thus we can construct a minimal annulus $A(\theta) \subset S(s, t)$ with convex Jordan curves boundary in $P_{s}$ and $P_{t}$ respectively, which enclose $\Sigma \cap P_{s}$ and $\Sigma \cap P_{t}$. Then by Lemma 2.1

$$
\left|\Gamma_{1}\right|+\left|\Gamma_{2}\right|>2(t-s), \quad\left|\Gamma_{1}\right|\left|\Gamma_{2}\right|>(t-s)^{2},
$$

where $\Gamma_{1}$ and $\Gamma_{2}$ are the projection of $\partial A(\theta)$. Note that we can make $\partial A(\theta)$ such that

$$
L(\theta, s)=\left|\Gamma_{1}\right|, \quad L(\theta, t)=\left|\Gamma_{2}\right| .
$$

Then all the conclusions are trivial by Lemma 2.1 .

Corollary 2.2. Let $c, d, \delta_{1}$, and $\delta_{2}$ be positive constant numbers. If $\Sigma$ is a connected compact non-planar minimal surface (maybe branched) such that $\partial \Sigma=L_{1} \cup L_{2}$ and

$$
L_{1} \subset D_{1}^{\prime}:=\left\{(x, y, z) ;-\delta_{1} \leq x-\frac{c}{d} z \leq \delta_{1}, z \leq 0\right\},
$$


Curvature estimates annuli and non-compact Douglas-Plateau problem 887

$$
L_{2} \subset D_{2}^{\prime}:=\left\{(x, y, z) ;-\delta_{2} \leq x-\frac{c}{d} z \leq \delta_{2}, z \geq d\right\},
$$

then

$$
2 \max \left\{\delta_{1}, \delta_{2}\right\}>\sqrt{c^{2}+d^{2}}
$$

Furthermore, if

$$
\begin{gathered}
L_{1} \subset D_{1}:=\left\{(x, y, 0) ;-\delta_{1} \leq x \leq \delta_{1}\right\}, \\
L_{2} \subset D_{2}:=\left\{(x, y, d) ;-\delta_{2} \leq x-c \leq \delta_{2}\right\}
\end{gathered}
$$

then

$$
\delta_{1}+\delta_{2}>\sqrt{c^{2}+d^{2}}
$$

Proof. Let $X: M \rightarrow \mathbb{R}^{3}$ be a conformal parametrization of $\Sigma$ and consider the function

$$
\phi=X_{1}-\frac{c}{d} X_{3}
$$

then $\phi$ is harmonic. Thus let $R:=\max \left\{\delta_{1}, \delta_{2}\right\}$, by maximum principle for harmonic functions,

$$
\Sigma \subset\left\{(x, y, z) ;-R \leq x-\frac{c}{d} z \leq R\right\} .
$$

Since $\partial \Sigma$ is contained in $\{z \leq 0\} \cup\{z \geq d\}, \Sigma \cap S(0, d)$ is a minimal surface whose boundary $L_{1}^{\prime} \cup L_{2}^{\prime}$ satisfies that

$$
L_{1}^{\prime} \subset\{(x, y, 0) ;-R \leq x \leq R\}, \quad L_{2}^{\prime} \subset\{(x, y, d) ;-R \leq x-c \leq R\} .
$$

Let $C_{1} \subset P_{0}, C_{2} \subset P_{d}$ be convex Jordan curves such that they enclose $L_{1}^{\prime}$ and $L_{2}^{\prime}$ respectively and

$$
C_{1} \subset\{(x, y, 0) ;-R \leq x \leq R\}, \quad C_{2} \subset\{(x, y, d) ;-R \leq x-c \leq R\} .
$$

By Theorem 2.2, there is a minimal annulus $A$ bounded by $C_{1}$ and $C_{2}$. Let $\pi$ be the $x z$-plane and $\Gamma_{i}, i=1,2,3,4$, be as defined in Lemma 2.1. By comparison principle for minimal surfaces, $\Gamma_{3}$ and $\Gamma_{4}$ are not line segments. Then by Lemma 2.1,

$$
4 R \geq\left|\Gamma_{1}\right|+\left|\Gamma_{2}\right| \geq\left|\Gamma_{3}\right|+\left|\Gamma_{4}\right|>\left|l_{3}\right|+\left|l_{4}\right|,
$$


where $l_{3}$ and $l_{4}$ are line segments such that $\Gamma_{1} \cup \Gamma_{2} \cup l_{3} \cup l_{4}$ consists of the boundary of a convex four-gon in the $x z$-plane. Elementary calculation shows that

$$
\left|l_{3}\right|+\left|l_{4}\right|=2 \sqrt{c^{2}+d^{2}} .
$$

Similarly we can prove (2.10) by requiring that

$$
C_{1} \subset\left\{(x, y, 0) ;-\delta_{1} \leq x \leq \delta_{1}\right\}, \quad C_{2} \subset\left\{(x, y, d) ;-\delta_{2} \leq x-c \leq \delta_{2}\right\} .
$$

Then we have

$$
2\left(\delta_{1}+\delta_{2}\right) \geq\left|\Gamma_{1}\right|+\left|\Gamma_{2}\right| \geq\left|\Gamma_{3}\right|+\left|\Gamma_{4}\right|>\left|l_{3}\right|+\left|l_{4}\right|,
$$

and

$$
\left|l_{3}\right|+\left|l_{4}\right|=\sqrt{\left(\delta_{2}-\delta_{1}+c\right)^{2}+d^{2}}+\sqrt{\left(\delta_{1}-\delta_{2}+c\right)^{2}+d^{2}} \geq 2 \sqrt{c^{2}+d^{2}} .
$$

To establish the existence of solutions to non-compact Douglas-Plateau problem with four parallel straight lines as boundary in Section 3, we need another curvature estimate for minimal annuli.

Proposition 2.2. Let $A \subset S\left(t_{1}, t_{2}\right) \cap S^{\prime}\left(t_{1}^{\prime}, t_{2}^{\prime}\right)$ be a compact embedded minimal annulus such that $\partial A=L_{1} \cup L_{2}$, where $L_{1} \subset P_{t_{1}}, L_{2} \subset P_{t_{2}}$ are $C^{2}$ convex Jordan curves. Let $E>0$ such that $|\kappa(p)| \leq E$ for any $p \in \partial A$, where $\kappa$ is the planar curvature. Then there is a constant $C_{1}>0$ only depending on $t_{2}-t_{1}, t_{2}^{\prime}-t_{1}^{\prime}$ and $E$, such that

$$
|K(p)| \leq C_{1},
$$

where $K(p)$ is the Gaussian curvature of $A$.

Proof. The proof is a generalization of the proof in [fanghwang-mr1] of a special case of this Proposition, the estimates in Lemma 2.1 enables us to make this generalization.

By a homothety, we can assume that $t_{1}=-1, t_{2}=1$. By a translation we can assume that $t_{1}^{\prime}=-R, t_{2}^{\prime}=R$ for some $R>0$.

If the Proposition is not true, then there are minimal annuli $B_{n} \subset$ $S(-1,1) \cap S^{\prime}(-R, R)$ such that $\partial B_{n}$ consists of two convex Jordan curves 
Curvature estimates annuli and non-compact Douglas-Plateau problem 889

in $P_{-1}$ and $P_{1}$, whose planar curvatures are bounded by $E$, and $\exists p_{n} \in B_{n}$ such that

$$
-C_{n}:=K_{B_{n}}\left(p_{n}\right) \leq K_{B_{n}}(p), \quad \forall p \in B_{n} ; \quad \lim _{n \rightarrow \infty} K_{B_{n}}\left(p_{n}\right)=-\infty .
$$

Let $\hat{B}_{n}=B_{n}-p_{n}:=\left\{p \in \mathbb{R}^{3}, p+p_{n} \in B_{n}\right\}$. Note that $p_{n}=\left(x_{n}, y_{n}, z_{n}\right)$, $-R \leq y_{n} \leq R,-1 \leq z_{n} \leq 1$. By a rotation if necessary, we may assume that $-1 \leq z_{n} \leq 0$. So that $\hat{B}_{n}=B_{n}-p_{n} \subset S(-1,2) \cap S^{\prime}(-2 R, 2 R)$ contains the origin, and $\partial \hat{B}_{n} \subset P_{-1-z_{n}} \cup P_{1-z_{n}}$.

Let $\tilde{B}_{n}=\sqrt{C_{n}} \hat{B}_{n}$ be the homothety of $\hat{B}_{n}$ and $K_{\tilde{B}_{n}}$ be the Gaussian curvature of $\tilde{B}_{n}$, then $\left|K_{\tilde{B}_{n}}\right| \leq 1$. Let $D_{m}$ be the ball centred at origin with radius $m$. Then by Lemma 2.3 a subsequence of $\left\{\tilde{B}_{n}\right\}$ converges in $D_{m}$. By a diagonal argument, a subsequence of $\left\{\tilde{B}_{n}\right\}$, still denote by $\left\{\tilde{B}_{n}\right\}$, converges to an embedded minimal surface $\tilde{M}$ in $\mathbb{R}^{3}$. $\tilde{M}$ is not a plane, since it has a point (the origin) with Gaussian curvature -1 .

Since the Gauss map $N: \tilde{B}_{n} \rightarrow S^{2}$ is one-to-one and $N \neq \pm(0,0,1)$, [fanghwang-mw1], we have $\int_{\tilde{B}_{n}} K_{\tilde{B}_{n}} d A>-4 \pi$, see [fanghwang-os1]. It forces that $\tilde{M}$ must have total curvature at least $-4 \pi$.

Since the boundaries of $\tilde{B}_{n}$ are on $P_{\sqrt{C_{n}}\left(-1-z_{n}\right)}$ and $P_{\sqrt{C_{n}}\left(1-z_{n}\right)}$ and $C_{n} \rightarrow$ $\infty$ as $n \rightarrow \infty$, if $\tilde{M}$ has a boundary, it must be that $\lim _{n \rightarrow \infty} \sqrt{C_{n}}\left(-1-z_{n}\right)$ exists in $\mathbb{R}$ or $\lim _{n \rightarrow \infty} \sqrt{C_{n}}\left(1-z_{n}\right)$ exists in $\mathbb{R}$. Since $-1 \leq z_{n} \leq 0$, it must be $\lim _{n \rightarrow \infty} \sqrt{C_{n}}\left(-1-z_{n}\right)=t_{0}$ exists and $\partial \tilde{M} \subset P_{t_{0}}$. Since $B_{n} \cap P_{t}$ are convex and have uniform planar curvature bound, $\partial \tilde{M}=\lim _{n \rightarrow \infty} \partial \tilde{B}_{n} \cap P_{\sqrt{C_{n}}\left(-1-z_{n}\right)}$ exists.

Since $\partial B_{n}$ has uniform planar curvature bound $E$, it turns out the planar curvature of $\partial \tilde{M}$ is bounded by $E / \sqrt{C_{n}} \rightarrow 0$, hence $\partial \tilde{M}$ must be a straight line $l$ if $\partial \tilde{M} \neq \emptyset$.

If $\partial \tilde{M} \neq \emptyset$, then rotating $\tilde{M}$ around $l=\partial \tilde{M}$ by $\pi$ degree, we get a complete minimal surface without boundary, its total curvature is at least $-8 \pi$, and it contains a straight line. But such a surface does not exist by classification, see for example [fanghwang-lo]. Thus $\partial \tilde{M}=\emptyset$.

It forces that $\tilde{M}$ must be a catenoid since that it is non-flat completely embedded without boundary, and its total curvature is at least $-4 \pi$.

Thus $\tilde{M} \cap P_{0}$ is a circle and since $\tilde{B}_{n} \rightarrow \tilde{M}$, the length of $\tilde{B}_{n} \cap P_{0}$ should be bounded, i.e., there is an $F>0$, such that

$$
\left|\tilde{B}_{n} \cap P_{0}\right| \leq F .
$$

But since $B_{n} \cap P_{t}$ is a convex Jordan curve for each $t \in(-1,1)$, and $B_{n} \cap$ $S(-1,1) \cap S^{\prime}(-R, R)$, recalling that $-1 \leq z_{n} \leq 0$ and applying (2.4) to 
$B_{n} \cap S\left(z_{n}, 1\right)$, we have

$$
\left|\tilde{B}_{n} \cap P_{0}\right|=\sqrt{C_{n}}\left|B_{n} \cap P_{z_{n}}\right|>\frac{\sqrt{C_{n}}\left(1-z_{n}\right)^{2}}{R} \geq \frac{\sqrt{C_{n}}}{R} \rightarrow \infty .
$$

Remark 2.2. Since we assume that $\partial A$ is $C^{2}$, the estimate is a global one, not just interior. But we can only prove by contradiction that the curvature of $A \subset S\left(t_{1}, t_{2}\right) \cap S^{\prime}\left(t_{1}^{\prime}, t_{2}^{\prime}\right)$ has an a prior bound. The ideal proof is to give an a prior estimate of curvature bound for $A \subset S\left(t_{1}, t_{2}\right) \cap S^{\prime}\left(t_{1}^{\prime}, t_{2}^{\prime}\right)$ explicitly involving the boundary planar curvature of $\partial A$, the height $t_{2}-t_{1}$, and the width $t_{2}^{\prime}-t_{1}^{\prime}$. Such a concrete estimate will be useful in many other cases.

Then we have another compactness theorem.

Theorem 2.3. If $\left\{A_{n}\right\} \subset S\left(t_{1}, t_{2}\right) \cap S^{\prime}\left(t_{1}^{\prime}, t_{2}^{\prime}\right)$ is a sequence of minimal annuli such that $\partial A_{n}=L_{1}^{n} \cup L_{2}^{n}$, where $L_{1}^{n} \subset P_{t_{1}}, L_{2}^{n} \subset P_{t_{2}}$ are $C^{2}$ convex Jordan curves with uniform planar curvature bound $E$ and $\lim _{n \rightarrow \infty} L_{1}^{n}=L_{1}$, $\lim _{n \rightarrow \infty} L_{2}^{n}=L_{2}$, then there is a subsequence of $\left\{A_{n}\right\}$ which converges to an embedded minimal surface $A$ such that $\partial A=L_{1} \cup L_{2}$.

Proof. Let $D_{r}$ be the ball centred at origin with radius $r$, then $\mathbb{R}^{3}=$ $\bigcup_{m=1}^{\infty} D_{m}$. Since $\lim _{n \rightarrow \infty} L_{1}^{n}=L_{1}, \lim _{n \rightarrow \infty} L_{2}^{n}=L_{2}$, for $m$ and $n$ large enough, $A_{n} \cap D_{m} \neq \emptyset$. By Proposition $2.2\left\{A_{n}\right\}$ has a uniform curvature bound, so Lemma 2.3 applied in $D_{m}$ gives a convergent subsequent $\left\{A_{m_{n}} \cap D_{m}\right\}$, then $\left\{A_{m_{m}}\right\}$ is a subsequence of $\left\{A_{n}\right\}$ which converges to an embedded minimal surface $A$ in any compact set. Since $\lim _{n \rightarrow \infty} L_{1}^{n}=L_{1}$, $\lim _{n \rightarrow \infty} L_{2}^{n}=L_{2}$, we have $\partial A=L_{1} \cup L_{2}$.

Remark 2.3. Note that the limit minimal surface $A$ is not necessarily an annulus. In fact, it may be even not connected.

\section{Applications to Non-Compact Douglas-Plateau Problem.}

First let us define various boundaries for which we want to solve the related Douglas-Plateau problem.

Let $\alpha: \mathbb{R} \rightarrow \mathbb{R}^{2}$ be a properly embedded complete convex curve, and let $L=\alpha(\mathbb{R})$. Suppose that $L$ is not a straight line, then $\mathbb{R}^{2}-L$ has two components, only one of them is convex. 
Curvature estimates annuli and non-compact Douglas-Plateau problem 891

Definition 3.1 (Standard Boundary). We call $\Gamma:=L_{1} \cup L_{2}$ a standard boundary if:

- $L_{1} \subset P_{-1}$ and $L_{2} \subset P_{1}$ are two continuously embedded, proper, complete, non-compact, non-flat convex curves.

- Let $Y_{1} \subset P_{-1}$ and $Y_{2} \subset P_{1}$ be the two convex domains bounded by $L_{1}$ and $L_{2}$ respectively. Let $\tilde{Y}_{i} \subset P_{0}$ and $\tilde{L}_{i} \subset P_{0}$ be the perpendicular projections of $Y_{i}$ and $L_{i}$, for $i=1,2$. Then $\tilde{Y}_{1} \cap \tilde{Y}_{2}$ is a bounded convex domain.

- There is a connected compact non-planar (maybe branched) minimal surface $\Sigma$ such that $\partial \Sigma \subset Y_{1} \cup Y_{2}$.

Remark 3.1. By Corollary 2.2, the last condition of Definition 3.1 implies that $\tilde{Y}_{1} \cap \tilde{Y}_{2} \neq \emptyset$. We will call this condition $\mathbf{H}-\mathbf{M}$ condition to $Y_{1} \cup Y_{2}$, it first appeared in [fanghwang-hm9] by Hoffman and Meeks.

Let $D_{r} \subset P_{0}$ be the disk centred at $(0,0)$ with radius $r$. It is well-known that if $r$ is large enough and $D_{r} \subset \tilde{Y}_{1} \cap \tilde{Y}_{2}$, then there is a piece of catenoid $C$ such that $\partial C \subset Y_{1} \cup Y_{2}$. Hence the H-M condition is satisfied.

Our first existence theorem is:

Theorem 3.1. Let $\Gamma$ be a standard boundary. Then there exist two embedded minimal annuli $\mathcal{A}$ and $\mathcal{B}$ such that $\partial \mathcal{A}=\partial \mathcal{B}=\Gamma$. The minimal annuli $\mathcal{A}$ and $\mathcal{B}$ have the following properties:

1. For each $t \in(-1,1), P_{t} \cap \mathcal{A}$ and $P_{t} \cap \mathcal{B}$ are strictly convex Jordan curves.

2. $\operatorname{Int}(\mathcal{A}) \cap \operatorname{Int}(\mathcal{B})=\emptyset$.

3. Let $N$ be a connected compact non-planar (maybe branched) minimal surface such that $\partial N \subset \bar{Y}_{1} \cup \bar{Y}_{2}$, then

$$
\operatorname{Int}(\mathcal{A}) \cap \operatorname{Int}(N)=\emptyset, \quad \mathcal{B} \cap N \neq \emptyset .
$$

4. $\mathcal{A}$ and $\mathcal{B}$ have the same symmetry groups as that of $\Gamma$. 
Remark 3.2. If we change the last condition in the definition of standard boundary so that $\partial \Sigma \subset \bar{Y}_{1} \cup \bar{Y}_{2}$, then there is at least one embedded minimal annulus $\mathcal{C}$ such that $\partial \mathcal{C}=\Gamma$. Furthermore, $\mathcal{C}$ behaves just like $\mathcal{A}$ in the sense that they satisfy the same properties in 1, 3 and 4 of Theorem 3.1.

A limit case of standard boundary is that of straight line boundary,

\section{Definition 3.2 (Straight Line Boundary). A straight line boundary} is as follows:

- $\Gamma=\bigcup_{i=1}^{4} L_{i}$, where $L_{i}$ are straight lines such that $L_{1}$ and $L_{2}$ are contained in $P_{-1}$ and parallel, while $L_{3}$ and $L_{4}$ are contained in $P_{1}$ and parallel. But $L_{1}$ and $L_{3}$ are not parallel.

- Let $Y_{1} \subset P_{-1}$ be the open strip bounded by $L_{1}$ and $L_{2}, Y_{2} \subset P_{1}$ be the open strip bounded by $L_{3}$ and $L_{4}$. Then $Y_{1} \cup Y_{2}$ satisfies the H-M condition.

Remark 3.3. Note that since $L_{1}$ and $L_{3}$ are not parallel, the perpendicular projections $\tilde{Y}_{1}$ and $\tilde{Y}_{2}$ of $Y_{1}$ and $Y_{2}$ have bounded intersection.

Theorem 3.2. Let $\Gamma$ be a straight line boundary. Then there exist two embedded minimal annuli $\mathcal{A}$ and $\mathcal{B}$ such that $\partial \mathcal{A}=\partial \mathcal{B}=\Gamma$. The minimal annuli $\mathcal{A}$ and $\mathcal{B}$ have all the properties stated in Theorem 3.1 .

Remark 3.4. The Remark 3.2 also applies to the straight line boundary.

Now let us consider parallel straight lines. It is also an limit case of a standard boundary. Indeed if we consider a standard boundary such that there is a straight line $L \subset P_{0}$ which intersects $\tilde{L}_{1}$ and $\tilde{L}_{2}$ in exactly one point respectively. We may change $L_{1}$ and $L_{2}$ such that the single intersection points of $L$ with $\tilde{L}_{1}$ and $\tilde{L}_{2}$ go to infinity in opposite directions, and $\tilde{L}_{1}$ and $\tilde{L}_{2}$ both break into two straight lines parallel to $L$. Thus we give the following definition of a parallel line boundary:

Definition 3.3 (Parallel Boundary). A parallel boundary is as follows:

- $\Gamma=\bigcup_{i=1}^{4} L_{i}$, where $L_{i}$ are parallel straight lines such that $L_{1}$ and $L_{2}$ are contained in $P_{-1}$, while $L_{3}$ and $L_{4}$ are contained in $P_{1}$. The strip bounded by $L_{1}$ and $L_{3}$ and the strip bounded by $L_{2}$ and $L_{4}$ are disjoint. 
Curvature estimates annuli and non-compact Douglas-Plateau problem 893

- Let $Y_{1} \subset P_{-1}$ be the open strip bounded by $L_{1}$ and $L_{2}, Y_{2} \subset P_{1}$ be the open strip bounded by $L_{3}$ and $L_{4}$ in $P_{1}$. Then $Y_{1} \cup Y_{2}$ satisfies the $\mathrm{H}-\mathrm{M}$ condition.

Remark 3.5. By Corollary 2.1, a necessary condition for $\Gamma=\bigcup_{i=1}^{4} L_{i}$ being a parallel boundary is that the product of the widths of the strips $Y_{1}$ and $Y_{2}$ is larger than 4 .

Given a parallel boundary $\Gamma$ as above, we always assume that the straight lines $L_{i}$ are parallel to the $x$-axis.

A special case of parallel boundary is a lattice boundary (it defines a lattice in the $y z$-plane):

Definition 3.4 (Lattice Boundary). Let $\Gamma$ be a parallel boundary. Let $p_{i}$ be the intersection points of the $L_{i}$ with the $y z$-plane. If the $p_{i}$ 's are the vertices of a parallelogram, then we call the parallel boundary a lattice boundary.

Let $F$ be the parallelogram with $p_{i}$ as vertices. Then we select the bisectrice point (the intersection of the two diagonals) of $F$ as the origin of $\mathbb{R}^{3}$.

Our third existence theorem is the following:

Theorem 3.3. Let $\Gamma$ be a parallel boundary, then there exists an embedded minimal annulus $\mathcal{D}$ such that $\partial \mathcal{D}=\Gamma$. Also $\mathcal{D}$ satisfies:

1. For $-1<t<1, P_{t} \cap \mathcal{D}$ are strictly convex Jordan curves.

2. Let $N$ be a connected compact non-planar (maybe branched) minimal surface such that $\partial N \subset \bar{Y}_{1} \cup \bar{Y}_{2}$, then $\mathcal{D} \cap N \neq \emptyset$.

3. $\mathcal{D}$ is invariant under the reflection $(x, y, z) \rightarrow(-x, y, z)$.

4. If $\Gamma$ is a lattice boundary, then $\mathcal{D}$ is invariant under the rotation of angle $\pi$ around the $x$-axis.

Remark 3.6. In [fanghwang-mr1], Meeks and Rosenberg gave a proof of the existence of $\mathcal{D}$ with a lattice boundary in order to construct doubly periodic minimal surfaces. 


\subsection{Proof of Theorem 3.1.}

The idea for the proof of Theorem 3.1 is to construct sequences of compact minimal annuli $\left\{A_{n}\right\}$ and $\left\{B_{n}\right\}$ whose convex boundaries approaching the given $\Gamma$.

Now we approach $L_{1}$ and $L_{2}$ by convex Jordan curves $L_{n}^{1} \subset P_{-1}$ and $L_{n}^{2} \subset P_{1}$ such that for any $R>0$, there is an $N_{R}>0$ such that whenever $n>N_{R}$,

$$
\left(L_{n}^{1} \cup L_{n}^{2}\right) \cap C_{R}=\left(L_{1} \cup L_{2}\right) \cap C_{R} .
$$

Let $D_{n}^{1}$ and $D_{n}^{2}$ be the disks bounded by $L_{n}^{1}$ and $L_{n}^{2}$. We can make $L_{n}^{1}$ and $L_{n}^{2}$ such that

$$
\partial \Sigma \subset D_{n}^{1} \cup D_{n}^{2}, \quad D_{n}^{1} \subset D_{n+1}^{1}, \quad D_{n}^{2} \subset D_{n+1}^{2},
$$

where $\Sigma$ is the surface in the H-M condition.

By Theorem 2.2 there are two minimal annuli $A_{n}$ and $B_{n}$, such that $\partial A_{n}=\partial B_{n}=L_{n}^{1} \cup L_{n}^{2}$.

To prove that there are convergent subsequences of $\left\{A_{n}\right\}$ and $\left\{B_{n}\right\}$, we need the following lemma. The proof of this technical lemma is quite involved. In order not to interrupt the main argument, at this moment let us assume the lemma is true.

First let us fix some more notations. Let $Q_{a}:=\left\{(x, y, z) \in \mathbb{R}^{3} ; x=a\right\}$, $H_{a}^{+}=\left\{(x, y, z) \in \mathbb{R}^{3} ; x \geq a\right\}, H_{a}^{-}=\left\{(x, y, z) \in \mathbb{R}^{3} ; x \leq a\right\}$. Let $W_{a}=$ $\{(x, y, z) ;-a \leq x \leq a\}$. Denote the $x z$-plane by $P_{0}^{\prime}$.

Lemma 3.1. Let $Y_{1}$ and $Y_{2}$ be as defined in Definition 3.1. Let $A \subset$ $S(-1,1)$ be a compact minimal surface. Suppose that $\partial A=C_{1} \cup C_{2}$ such that $C_{1} \subset Y_{1}, C_{2} \subset Y_{2}$. Then we can choose coordinates $(x, y, z)$ such that for any $a>0$, there is an $S(a)>0$ such that $A \cap W_{a} \subset S^{\prime}(-S(a), S(a))$. We can choose that if $a>b>0$, then $S(a) \geq S(b)$.

And for any $t \in(-1,1)$, there is an $R(t)>0$ such that

$$
A \cap P_{t} \subset C_{R(t)}
$$

Moreover, we can make that $R(t)=R(-t)$ and $R(s) \leq R(t)$ whenever $|s|<|t|$.

As proved in the proof of Lemma 3.1, we can choose coordinates $(x, y, z)$ of $\mathbb{R}^{3}$, such that if $a>0$ is large enough, we have $Q_{a} \cap L_{2}=\emptyset$ and $Q_{-a} \cap L_{1}=$ 
Curvature estimates annuli and non-compact Douglas-Plateau problem 895

$\emptyset$ or, $Q_{a} \cap L_{1}=\emptyset$ and $Q_{-a} \cap L_{2}=\emptyset$, but not both. Without loss of generality, we assume that it is the former and keep this convention in this paper.

By Lemma 3.1, $A_{n} \cap S(-t, t) \subset C_{R(t)}$ and $B_{n} \cap S(-t, t) \subset C_{R(t)}$, hence for each $t \in(0,1)$, they are uniformly bounded. By Shiffman's first theorem [fanghwang-sh1], $A_{n} \cap P_{t}$ and $B_{n} \cap P_{t}$ are strictly convex Jordan curves.

Now we can use Theorem 2.1 to prove subsequences of $\left\{A_{n}\right\}$ and $\left\{B_{n}\right\}$ converge to $\mathcal{A}$ and $\mathcal{B}$ in the interior of $S(-1,1)$. In fact, there are subsequences of $\left\{A_{n}\right\}$ and $\left\{B_{n}\right\}$ which are convergent to embedded compact minimal annuli $A_{t_{m}} \subset S\left(-t_{m}, t_{m}\right)$ and $B_{t_{m}} \subset S\left(-t_{m}, t_{m}\right)$ in $S\left(-t_{m}, t_{m}\right)$ for any $t_{m}$, where $t_{m} \nearrow 1$ as $m \rightarrow \infty$. By a diagonal argument we see that subsequences of $\left\{A_{n}\right\}$ and $\left\{B_{n}\right\}$ converge to embedded minimal surfaces $\mathcal{A}$ and $\mathcal{B}$. Since for each $s \in\left(-t_{m}, t_{m}\right), A_{n} \cap P_{s}$ and $B_{n} \cap P_{s} \subset C_{R\left(t_{m}\right)}$ is uniformly bounded convex Jordan curves and the convergence is smooth, $\mathcal{A} \cap P_{s}$ and $\mathcal{B} \cap P_{s}$ are convex Jordan curves. Since for $t \in(-1,1), \mathcal{A} \cap P_{t}$ and $\mathcal{B} \cap P_{t}$ are convex, by Shiffman's theorem again, we know that $\mathcal{A} \cap P_{t}$ and $\mathcal{B} \cap P_{t}$ are strictly convex, hence $\mathcal{A}$ and $\mathcal{B}$ are minimal annuli.

Still denote these subsequences by $\left\{A_{n}\right\}$ and $\left\{B_{n}\right\}$, we only need prove that $\mathcal{A}$ and $\mathcal{B}$ are continuous up to boundary and $\partial \mathcal{A}=\partial \mathcal{B}=\Gamma$.

Now by Lemma $2.1 A_{n}$ consists of two simply connected minimal graphs over a domain $\Omega_{n} \subset P_{0}^{\prime}$, say $G_{n}^{+}, G_{n}^{-}$.

Since $A_{n}=G_{n}^{+} \cup G_{n}^{-}, A_{n} \cap Q_{a}$ and $A_{n} \cap Q_{-a}$ are the unions of two graphs respectively, hence they are simple curves. Similarly, $\mathcal{A} \cap Q_{a}$ and $\mathcal{A} \cap Q_{-a}$ are simple curves. Thus $G_{n}^{+} \cap W_{a}, G_{n}^{-} \cap W_{a}, G^{+} \cap W_{a}$, and $G^{-} \cap W_{a}$ are all simply connected.

Let $\Omega_{a}=\Omega \cap W_{a}$, then $\Omega_{a}$ is bounded and has piecewise smooth boundary as proved in Lemma 2.1, hence $\partial \Omega_{a}$ has finite length. Also by Lemma 3.1, $\mathcal{A} \cap W_{a}$ is also bounded, thus we know that $G^{+} \cap W_{a}, G^{-} \cap W_{a}$ have finite area.

Let $D$ be the closed unit disk in $\mathbb{C}$ and $X_{n}: D \rightarrow \mathbb{R}^{3}$ be a conformal embedding of $G_{n}^{+} \cap W_{a}$ such that for three fixed points $p_{i} \in \partial D, X_{n}\left(p_{i}\right)=q_{i}$, where $q_{i} \in \partial G_{n}^{+} \cap\left(L_{1} \cup L_{2}\right) \cap W_{a}, i=1$, 2, 3. Since $\partial A_{n} \rightarrow L_{1} \cup L_{2}$, this is alway possible. Since $G_{n}^{+} \rightarrow G^{+}$, the areas of $G_{n}^{+} \cap W_{a}$ are uniformly bounded and by the conformality of $X_{n}, \int_{D}\left|D X_{n}\right|^{2}$ are uniformly bounded.

By Courant-Lebesgue Lemma, $G_{n}^{+} \cap W_{a}=X_{n}(D)$ converges to $\left(G^{+} \cup\right.$ $\left.\left(L_{1} \cup L_{2}\right)\right) \cap W_{a}$ and is continuous up to boundary. Similar argument for $G^{-}$ also holds. Thus we see that $\partial\left(\mathcal{A} \cap W_{a}\right) \cap\left(P_{-1} \cup P_{1}\right)=\left(L_{1} \cup L_{2}\right) \cap W_{a}$ for all $a>0$ large enough. Moreover, it is clear that $\partial \mathcal{A} \subset P_{-1} \cup P_{1}$. Therefore $\partial \mathcal{A}=L_{1} \cup L_{2}$ and similarly $\partial \mathcal{B}=L_{1} \cup L_{2}$ and they are continuous up to boundary.

Let $N$ be a connected non-planar compact (maybe branched) minimal 
surface such that $\partial N \subset \bar{Y}_{1} \cup \bar{Y}_{2}$. Let $V_{n}$ be the solid bounded by $A_{n} \cup D_{n}^{1} \cup$ $D_{n}^{2}$, and $V$ be the solid bounded by $\mathcal{A} \cup \bar{Y}_{1} \cup \bar{Y}_{2}$. We know that $N \subset V_{n}$ and $\operatorname{Int}\left(A_{n}\right) \cap \operatorname{Int}(N)=\emptyset$. Since $V_{n} \rightarrow V, N \subset V$. By the comparison principle for minimal surfaces, either $\mathcal{A}=N \operatorname{or} \operatorname{Int}(\mathcal{A}) \cap \operatorname{Int}(N)=\emptyset$. Since $N$ is compact and $\mathcal{A}$ is not compact, $\operatorname{Int}(N) \cap \operatorname{Int}(\mathcal{A})=\emptyset$. In particular, $\operatorname{Int}(\Sigma) \cap \operatorname{Int}(\mathcal{A})=\emptyset$

Since $B_{n} \cap N \neq \emptyset, \lim _{n \rightarrow \infty} B_{n}=\mathcal{B}$, and $N$ is compact, we know that $\mathcal{B} \cap N \overline{;} \neq \emptyset$.

In particular, $\operatorname{Int}(\Sigma) \cap \operatorname{Int}(\mathcal{B}) \neq \emptyset$. Thus $\mathcal{A} \neq \mathcal{B}$.

Let $V_{n}^{\prime}$ be the solid bounded by $B_{n} \cup D_{n}^{1} \cup D_{n}^{2}$, and $V^{\prime}$ be the solid bounded by $\mathcal{B} \cup \bar{Y}_{1} \cup \bar{Y}_{2}$. Then since $V_{n}^{\prime} \subset V_{n}, \lim _{n \rightarrow \infty} V_{n}=V$, and $\lim _{n \rightarrow \infty} V_{n}^{\prime}=V^{\prime}$, $V^{\prime} \subset V$. By the comparison principle for minimal surfaces, we have that $\operatorname{Int}(\mathcal{A}) \cap \operatorname{Int}(\mathcal{B})=\emptyset$.

By Theorem 2.2, we can construct the approaching sequences $\left\{A_{n}\right\}$ and $\left\{B_{n}\right\}$ such that they have the same symmetry groups as that of $\Gamma$, thus the limits, $\mathcal{A}$ and $\mathcal{B}$, have the same symmetry groups as that of $\Gamma$.

The proof of Theorem 3.1 is complete except that we still need prove Lemma 3.1.

The idea for the proof of Lemma 3.1 is to construct various barriers and use the comparison principle for minimal surfaces. To establish these barriers, let us quote a Lemma in [fanghwang-chm].

Lemma 3.2 (Lemma 4 in [fanghwang-chm]). Let $L_{0}$ consist of two non-collinear rays emanating from the origin in the plane $P_{0}$, and let $L_{1}$ be their vertical translation into the plane $P_{1}$. Then $L_{0} \cup L_{1}$ is the boundary of a unique properly embedded minimal surface contained in the convex hull of $L_{0} \cup L_{1}$. This minimal surfaces is a graph over an infinite strip and hence is simply connected.

Remark 3.7. As pointed out in the proof of Lemma 3.2 in [fanghwang$\mathrm{chm}], A$ is asymptotic to a flat strip as it diverges to infinity.

Proof of Lemma 3.1. First we claim that $\tilde{Y}_{1} \cup \tilde{Y}_{2}$ is contained in an unbounded domain $\Omega$ with four rays as boundary. And if we adjust the angles between the boundary rays of $\Omega$ we can assume that there is a straight line $l$ contained in $\Omega$. Note that this implies that $\mathbb{R}^{3}-\bar{\Omega}$ consists of two unbounded convex domains.

In fact, since $\tilde{Y}_{1} \cap \tilde{Y}_{2}$ is convex and bounded, there are exactly two unbounded components on $\tilde{L}_{1}-\tilde{L}_{2}$ and $\tilde{L}_{2}-\tilde{L}_{1}$. Say $\alpha_{i} \subset \tilde{L}_{1}-\tilde{L}_{2}, \beta_{i} \subset$ 
Curvature estimates annuli and non-compact Douglas-Plateau problem 897

$\tilde{L}_{1}-\tilde{L}_{2}, i=1,2$, are the four unbounded components. Take a point on each of these unbounded components, say $p \in \alpha_{1}, p^{\prime} \in \alpha_{2}, q \in \beta_{1}, q^{\prime} \in \beta_{2}$. Since $\tilde{L}_{i}$ are convex, there are straight lines passing through these four points such that $\tilde{L}_{i}$ are on the same side of these lines. Denote these lines by $l_{p}, l_{p^{\prime}}, l_{q}$, and $l_{q^{\prime}}$.

If $l_{p}$ and $l_{p^{\prime}}$ interset, then $\tilde{Y}_{1}$ is contained in a wedge $\Omega_{1}$ (a convex domain bounded by two rays issuing from one point) bounded by rays in $l_{p}$ and $l_{p^{\prime}}$. If $l_{p}$ and $l_{p^{\prime}}$ are parallel, then since $\tilde{L}_{1}$ is non-compact convex, $\tilde{Y}_{1}$ is contained in the strip $S$ bounded by $l_{p}$ and $l_{p^{\prime}}$. Since $\tilde{L}_{1}$ is non-flat, we can find a wedge $\Omega_{1}$ such that $\tilde{Y}_{1} \subset \Omega_{1}$. Similarly, there is a wedge $\Omega_{2} \supset \tilde{Y}_{2}$.

Since $\tilde{Y}_{1} \cap \tilde{Y}_{2}$ is compact, by parallel translations or, if necessary, vary the angles of the wedges, we can assume that $\partial \Omega_{1} \cap \partial \Omega_{2}=\{P, Q\}$. Take $\Omega$ to be the domain bounded by rays in $\partial \Omega_{1} \cup \partial \Omega_{2}$ issuing from $P$ and $Q$, then clearly $\tilde{Y}_{1} \cup \tilde{Y}_{2} \subset \Omega$. By enlarging $\Omega$ if necessary, we can assume that the straight line $l$ which is the bisector of the line segment $\overline{P Q}$ is contained in $\Omega$. Take $l$ as the $x$-axis, then the coordinate system of $(x, y, z)$ satisfies that if $a>0$ large enough, we have $Q_{a} \cap L_{2}=\emptyset$ and $Q_{-a} \cap L_{1}=\emptyset$ or, $Q_{a} \cap L_{1}=\emptyset$ and $Q_{-a} \cap L_{2}=\emptyset$, but not both.

Denote the two components of $\partial \Omega$ by $l^{1}$ and $l^{2}$.

Now let $A \subset S(-1,1)$ be a compact minimal surface such that $\partial A=$ $C_{1} \cup C_{2}, C_{1} \subset Y_{1}, C_{2} \subset Y_{2}$. Then $C_{1} \cap L_{2}=\emptyset$ and $C_{2} \cap L_{1}=\emptyset$. See Figure 3 below.

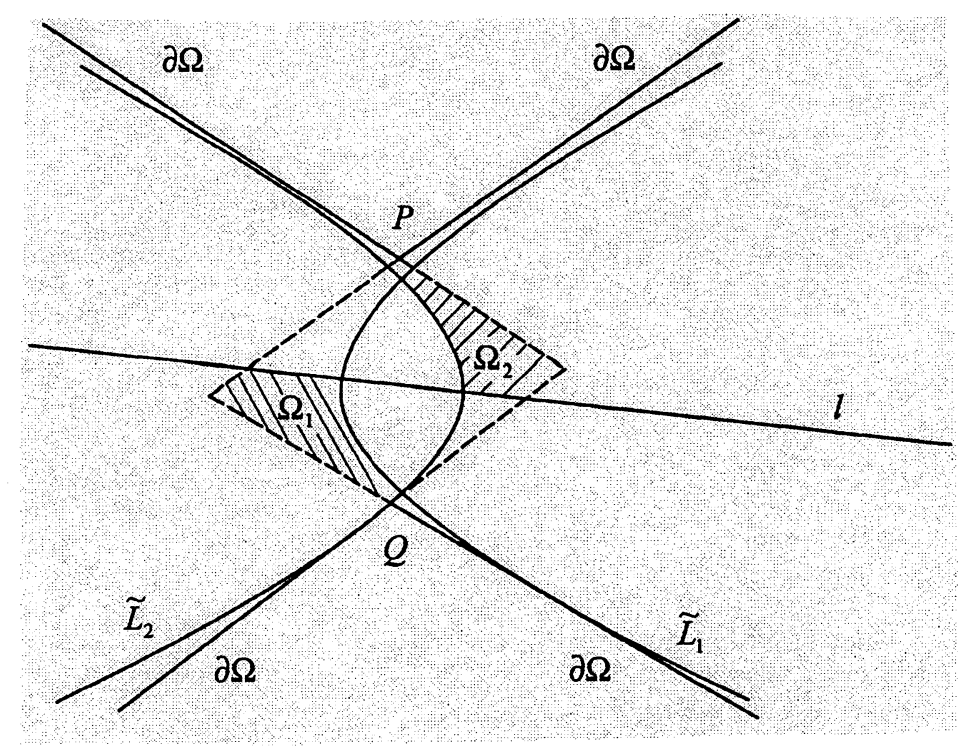

Figure 3 
We first prove that for any $a>0$, there is an $S(a)>0$ such that $A \cap W_{a} \subset S^{\prime}(-S(a), S(a))$.

We use the barrier in Lemma 3.2.

$$
l^{1} \cap\left(\tilde{Y}_{1} \cup \tilde{Y}_{2}\right)=\emptyset .
$$

Now let us parallel translate $l^{1}$ along the $z$-axis into $P_{-1}$ and $P_{1}$ and call them $l_{-1}^{1}$ and $l_{1}^{1}$ respectively. By Lemma 3.2 , there is a minimal graph $M$ bounded by $l_{-1}^{1}$ and $l_{1}^{1}$. By (3.3), $\partial A \cap\left(l_{-1}^{1} \cup l_{1}^{1}\right)=\emptyset$. By the comparison principle for minimal surfaces, $A \cap M=\emptyset$. Thus for any $a \neq 0$, there is an $R_{a}^{1}>0$, such that $A \cap W_{a}$ is contained in the half-space $\left\{y \leq R_{a}^{1}\right\}$. Since $M$ is continuous, we can select $R_{a}^{1}$ such that it is nondecreasingly continuous respect to $a$.

Similarly, using $l^{2}$ to make minimal graph $M^{\prime}$, we can find an $R_{a}^{2}>0$ such that $\mathcal{P}(A) \cap W_{a}$ is contained in the half-space $\left\{y \geq-R_{a}^{2}\right\}$ and $R_{a}^{2}$ is nondecreasingly continuous respect to $a$. Take $S(a)=\max \left\{R_{a}^{1}, R_{a}^{2}\right\}$, we have proved that $A \cap W_{a} \subset S^{\prime}(-S(a), S(a))$ and $S(a)$ is nondecreasingly continuous respect to $a$.

Next, the minimal graph $M$ bounded by $l_{-1}^{1} \cup l_{1}^{1}$ is contained in the convex hull of $l_{-1}^{1} \cup l_{1}^{1}$. Let $\mathcal{P}$ be the perpendicular projection on the $x y$-plane. Then $\mathcal{P}(M)$ is contained in the convex domain bounded by $l^{1}$. Similarly, $\mathcal{P}\left(M^{\prime}\right)$ is contained in the convex domain bounded by $l^{2}$. Therefore, $\mathcal{P}(M) \cap\left(\tilde{Y}_{1} \cup\right.$ $\left.\tilde{Y}_{2}\right)=\emptyset$ and $\mathcal{P}\left(M^{\prime}\right) \cap\left(\tilde{Y}_{1} \cup \tilde{Y}_{2}\right)=\emptyset$. Parallel moving $l^{1}$ and $l^{2}$ to $L^{1}$ and $L^{2}$ along the $\pm y$-direction and denote the non-convex domain bounded by $L^{1} \cup L^{2}$ by $\Omega^{\prime}$, then by the Remark 3.7 we can make

$$
\left(\mathcal{P}(M) \cup \mathcal{P}\left(M^{\prime}\right)\right) \cap \Omega^{\prime}=\emptyset, \quad \mathcal{P}(A) \subset \Omega^{\prime} .
$$

Still denote $L^{1}$ by $l^{1}, L^{2}$ by $l^{2}$.

Let $l_{u}=\{(x, y, z) ; x=u, z=0\}$. We have proved and made the convention that $\tilde{L}_{1} \cap l_{-d}=\emptyset, \tilde{L}_{2} \cap l_{d}=\emptyset$ for $d>0$ large enough.

Let $\Omega_{1}$ be the unbounded convex domain bounded by part of $l^{1} \cup l^{2} \cup l_{d}$. Then $\partial \Omega_{1}=\Gamma_{1} \cup \Gamma_{2}$, where $\Gamma_{1} \subset l^{1} \cup l^{2}$ consists of two rays issued from the intersections of $l_{d}$ with $l^{1} \cup l^{2}$, and $\Gamma_{2}$ is the line segment in $l_{d}$ between the two intersection points.

Similarly, let $\Omega_{2}$ be the unbounded convex domain bounded by part of $l^{1} \cup l^{2} \cup l_{-d}$. Then $\partial \Omega_{2}=\Gamma_{3} \cup \Gamma_{4}$, where $\Gamma_{3} \subset l^{1} \cup l^{2}$ consists of two rays issued from the intersections of $l_{-d}$ with $l^{1} \cup l^{2}$, and $\Gamma_{4} \subset l_{-d}$ is a line segment. See Figure 4 below.

We construct two minimal graphs $G_{1}$ and $G_{2}$ by solving Dirichlet problems for minimal surface equation on the domains $\Omega_{1}$ and $\Omega_{2}$, with the 
Curvature estimates annuli and non-compact Douglas-Plateau problem 899 boundary data:

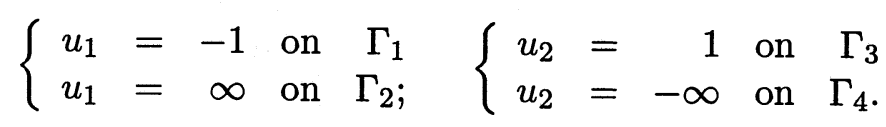

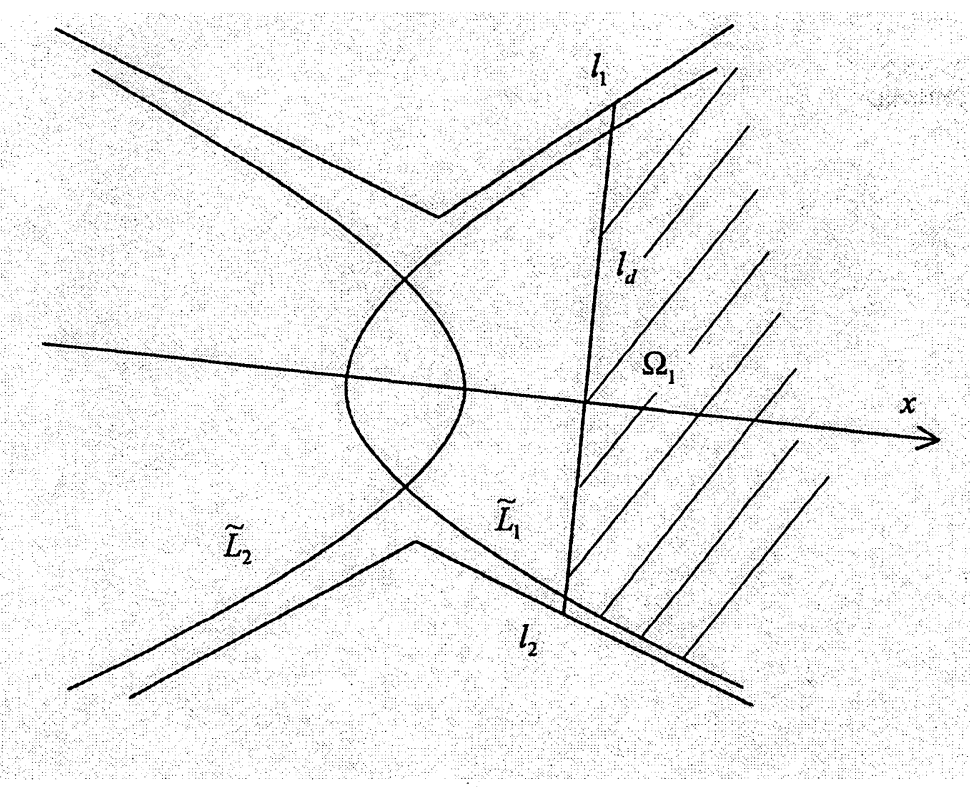

Figure 4

Such minimal graphs exist and are unique. For example, let $\Omega_{a}$ be the domain $\left\{(x, y) \in \Omega_{1} ; x<a\right\}$, then $\partial \Omega_{a}=\Gamma_{2} \cup \Gamma_{a} \cup \Gamma_{1}^{a}$, where $\Gamma_{a}$ is the segment in $l_{a}$ between the intersection points of $l_{a}$ with $\Gamma_{1} ; \Gamma_{1}^{a}$ is $\Gamma_{1} \cap\{x<a\}$. Then clearly when $a$ large enough, we have

$$
\left|\Gamma_{1}^{a}\right|>\left|\Gamma_{2}\right|+\left|\Gamma_{a}\right| .
$$

By a theorem of Jenkins and Serrin [fanghwang-js], there is a unique solution $u_{a}$ for the Dirichlet problem of minimal surface equation with the boundary value:

$$
\begin{aligned}
& u_{a}=-1 \text { on } \Gamma_{1}^{a} \\
& u_{a}=\infty \text { on } \Gamma_{2} \cup \Gamma_{a} .
\end{aligned}
$$


Now if $c>a$, then by comparison principle for minimal surfaces, $u_{c}<u_{a}$ in $\Omega_{a}$. For any $p \in \Omega_{1}$, if $a$ is large enough then $p \in \Omega_{a}$. Let $c_{n} \nearrow \infty$ then $\left\{u_{c_{n}}\right\}$ is a decreasing sequence, by the monotone convergence theorem [fanghwangjs], there is a $u=\lim _{n \rightarrow \infty} u_{c_{n}}$ which solves our Dirichlet problem. By the maximum principle in [fanghwang-ni1], page 256, $\sup _{x>d+1}\left|u_{1}(x, y)\right|$ and $\sup _{x \leq-d-1}\left|u_{2}(x, y)\right|$ are finite. Hence by Theorem 3 of [fanghwang-hwa1], the solution is unique.

An important property for $G_{i}$ is that $\lim _{|(x, y)| \rightarrow \infty} u_{i}(x, y)=\mp 1, i=1$, 2, see [fanghwang-mr2], Theorem 3.1. Thus by (3.4), for any $t \in(-1,1)$, there is an $R_{1}(t)>0$ such that

$$
G_{1} \cap P_{t} \subset H_{R_{1}(t)}^{-} ; \quad G_{2} \cap P_{t} \subset H_{-R_{1}(t)}^{+} .
$$

By the construction of $G_{i}, \partial A \cap \partial G_{i}=\emptyset$ and $\mathcal{P}\left(A \cap H_{d}^{+}\right) \subset \mathcal{P}\left(G_{1}\right)=\Omega_{1}$, $\mathcal{P}\left(A \cap H_{-d}^{-}\right) \subset \mathcal{P}\left(G_{2}\right)=\Omega_{2}$. By the comparison principle for minimal surfaces, we conclude that $A \cap G_{i}=\emptyset$, for $i=1$, 2. Since $\mathcal{P}(A) \cap\left(\tilde{Y}_{1} \cap \tilde{Y}_{2}\right) \neq \emptyset$ and $\mathcal{P}\left(G_{i}\right) \cap\left(\tilde{Y}_{1} \cap \tilde{Y}_{2}\right)=\emptyset, i=1,2$, by the maximum principle $A \cap P_{t} \subset$ $H_{R_{1}(t)}^{-} \cap H_{-R_{1}(t)}^{+}, R_{1}(t) \geq d$, for any $t \in(-1,1)$. Then we have $S\left(R_{1}(t)\right)>0$, such that $A \cap P_{t} \subset W_{R_{1}(t)} \cap S^{\prime}\left(-S\left(R_{1}(t)\right), S\left(R_{1}(t)\right)\right.$.

Take $R(t)=\max \left\{\sqrt{2} R_{1}(t), \sqrt{2} S\left(R_{1}(t)\right)\right\}$, then $A \cap P_{t} \subset C_{R(t)}$. Since $G_{1}$ and $G_{2}$ are continuous, $R_{1}(t)$ is nondecreasingly continuous respect to $t$. Thus $R(t)$ is nondecreasingly continuous respect to $t$.

The proof of Lemma 3.1 is complete.

\subsection{Proof of Theorem 3.2 .}

The proof of Theorem 3.2 is similar to the proof of Theorem 3.1. The key point is that we can confine approaching minimal annulus sequences $\left\{A_{n}\right\}$ and $\left\{B_{n}\right\}$ by four minimal barriers, i.e., the minimal graphs as in Lemma 3.2 , using the eight rays issuing form the four intersection points of $\tilde{L}_{i}, i=1$, $2,3,4$. Thus Lemma 3.1 is true for a straight line case. The other arguments are either exactly the same as the arguments in the proof of Theorem 3.1 or are slightly variations of them.

\subsection{Proof of Theorem 3.3.}

We start with $L_{n}^{1}$ being the convex curve consisting of the two line segments $\left(L_{1} \cup L_{2}\right) \cap C_{n}$, and two round arcs smoothly connecting the two pairs of end points, note that we use the same arcs up to a translation or reflection. Similarly define $L_{n}^{2}$. By this construction, $L_{n}^{1}$ and $L_{n}^{2}$ are invariant under the 
Curvature estimates annuli and non-compact Douglas-Plateau problem 901

reflection about the $y z$-plane and have uniformly bounded boundary planar curvature.

If $\Gamma$ is a lattice boundary, then we can make $L_{n}^{2}$ to be the image of $L_{n}^{1}$ under the rotation of angle $\pi$ around the $x$-axis.

By Theorem 2.2, there are embedded minimal annuli $B_{n}$ bounded by $L_{n}^{1} \cup L_{n}^{2}$ such that $\operatorname{Int}\left(B_{n}\right) \cap \operatorname{Int}(\Sigma) \neq \emptyset$, where $\Sigma$ is the minimal surface in the $\mathrm{H}-\mathrm{M}$ condition of Definition 3.3. Moreover, $B_{n}$ has the same symmetry group as that of $L_{n}^{1} \cup L_{n}^{2}$. Note that there is an $R>0$ such that $B_{n} \subset S^{\prime}(-R, R)$ and by the construction, we see that $L_{n}^{1} \cup L_{n}^{2}$ have uniform boundary planar curvature bound and $\lim _{n \rightarrow \infty} L_{n}^{1} \cup L_{n}^{2}=\Gamma$. Hence we can apply Theorem 2.3 to conclude that there is a subsequence of $\left\{B_{n}\right\}$ which converges to an embedded minimal surface $\mathcal{D}$ such that $\partial \mathcal{D}=\Gamma$.

Since $B_{n}$ 's satisfy the symmetry conditions in Theorem $3.3, \mathcal{D}$ also satisfies the symmetry conditions.

It remains to prove that $\mathcal{D}$ is an annulus and satisfies the other properties claimed in Theorem 3.3.

To establish that $\mathcal{D}$ is an annulus, it is sufficient to prove that $\mathcal{D} \cap P_{t}$ is a strictly convex Jordan curve, for any $-1<t<1$.

Since $B_{n} \cap \Sigma \neq \emptyset$ and $\Sigma$ is compact, we see that $\mathcal{D} \cap \Sigma \neq \emptyset$.

We observe that $P_{t} \cap \mathcal{D}$ is the smooth limit of a sequence of strictly convex Jordan curves. If $P_{t} \cap \mathcal{D}$ is compact, then it must be a convex Jordan curve. Thus the only thing left to be proved is that $\mathcal{D} \cap P_{t}$ must be compact.

Note that $P_{t} \cap \mathcal{D}$ is invariant under the reflection about the $y z$-plane. If $P_{t} \cap \mathcal{D}$ is not compact, then clearly $P_{t} \cap \mathcal{D} \cap\{x= \pm s\} \neq \emptyset$, for any $s>0$, otherwise $P_{t} \cap \mathcal{D}$ is bounded. This forces that $P_{t} \cap \mathcal{D}$ consists of two graphs generated by uniformly bounded functions $y_{1}(x, t)$ and $y_{2}(x, t)$, $y_{1} \geq y_{2},-\infty<x<\infty$, or in the limit case, $y_{1} \equiv y_{2}$. Let $y_{1}^{n}$ and $y_{2}^{n}$ be the functions defined by $P_{t} \cap B_{n}$, then $y_{1}^{n}$ is concave and $y_{2}^{n}$ is convex. Since $y_{1}(x)=\lim _{n \rightarrow \infty} y_{1}^{n}, y_{2}(x)=\lim _{n \rightarrow \infty} y_{2}^{n}$, in $C^{k}$ topology, $\forall k>0$, $y_{i}^{n}(x)=y_{i}^{n}(-x), i=1,2, y_{1}$ is concave with maximum $y_{1}(0, t)$ and $y_{2}$ is convex with minimum $y_{2}(0, t)$. By Lemma 2.1

$$
\left|y_{1}(0, t)-y_{2}(0, t)\right|>\max \left\{\frac{(1-t)^{2}}{4 R^{2}}, \frac{(t+1)^{2}}{4 R^{2}}\right\} \geq \frac{1}{4 R^{2}},
$$

for $-1<t<1$. If $P_{t} \cap \mathcal{D}$ is not compact, then $y_{1}$ and $y_{2}$ are both defined on $(-\infty, \infty)$, and $y_{1}$ is concave, $y_{2}$ is convex. Thus $P_{t} \cap \mathcal{D}$ is the union of two parallel straight lines which are parallel to the $x$-axis.

Consider the arc length functions $L(t)=$ the arc length of $P_{t} \cap \mathcal{D}, \quad$ and $\quad L_{n}(t)=$ the arc length of $P_{t} \cap B_{n}$. 
Then $L_{n}(t) \rightarrow L(t)$ when $n \rightarrow \infty$. By [fanghwang-osh], $L_{n}$ is convex with respect to $t$. We see that if for some $-1<t_{0}<1, L\left(t_{0}\right)=\infty$, then $L_{n}\left(t_{0}\right) \rightarrow \infty$ and there is a closed interval containing $t_{0}$ in $(-1,1)$ such that on which $L_{n}(t) \rightarrow \infty$. Thus we can assume that the set

$$
\left\{t \in(-1,1) \mid P_{t} \cap \mathcal{D} \text { is not compact }\right\}
$$

contains an open interval in $(-1,1)$. Hence there are $-1<t_{1}<t_{2}<1$ such that $P_{t} \cap \mathcal{D}$ consists of two straight lines parallel to the $x$-axis for $t_{1}<t<t_{2}$. Thus $\mathcal{D}$ is contained in a ruled minimal surface. Since the only non-planar ruled minimal surface is the Helicoid and its generating straight lines are not parallel, $\mathcal{D}$ is contained in two planes $P_{1}$ and $P_{2}$ such that $L_{1} \cup L_{2} \cup L_{3} \cup L_{4} \subset P_{1} \cup P_{2}$. Now since $\mathcal{D}$ is embedded, we have that $P_{1}$ is the plane containing $L_{1} \cup L_{3}, P_{2}$ is the plane containing $L_{2} \cup L_{4}$. But by the comparison principle for minimal surfaces, $\Sigma \cap\left(P_{1} \cup P_{2}\right)=\emptyset$, and $\Sigma \cap \mathcal{D}=\emptyset$, a contradiction. This contradiction proves that $P_{t} \cap \mathcal{D}$ is compact.

As before, once we know that $P_{t} \cap \mathcal{D}$ is convex for $-1<t<1$, then it is strictly convex by quoting Shiffman's first theorem.

The remaining properties claimed in Theorem 3.3 can be proved in the same way as in the proof of Theorem 3.1.

The proof of Theorem 3.3 is complete now.

Remark 3.8. There are other cases of boundaries such that similar lemma as Lemma 3.1 is true, thus with H-M condition, there are two solutions. For example, $L_{1}$ becomes two parallel straight lines and $\tilde{Y}_{1} \cap \tilde{Y}_{2}$ is bounded, etc.

Is the solution in Theorem 3.3 unique? Similar questions can be asked. For example, are there other solutions besides the two given in Theorem 3.1 and 3.2? is there a theory about non-compact smooth convex boundary as that established by Meeks and White in [fanghwang-mw1]? Furthermore, can the theory of Meeks and White, together with its generalization to noncompact cases (if it is generalizable), be generalized to the continuous case? We would like to know the answers.

\section{References.}

[1] M. Anderson, Curvature estimates for minimal surfaces in 3-manifolds, Ann. scient. Éc. Norm. Sup. 18 (1985), 89-105.

[2] M. Callahan, D. Hoffman, and W. Meeks III, The structure of singly-periodic minimal surfaces, Invent. math. 99 (1990), 455-81. 
Curvature estimates annuli and non-compact Douglas-Plateau problem 903

[3] U. Dierkes, S. Hildebrandt, A. Küster, and O. Wohlrab, Minimal Surfaces I, Springer-Verlag 1992.

[4] J. Douglas, The problem of Plateau for two contours, J. Math. Phys. Massachusetts Inst. of Tech. 10 (1930/31), 315-59.

[5] Y. Fang, Minimal annuli in $\mathbf{R}^{3}$ bounded by non-compact complete convex curves in parallel planes, J. of Austral. Math. Soc. (Series A) 60 (1996), 36988.

[6] D. Hoffman and W. Meeks III, A variational approach to the existence of complete embedded minimal surfaces, Duke Mathematical Journal, 57 (1988), $877-93$.

[7] J. Hwang, A uniqueness theorem for the minimal surface equation, Pacific J. of Mathematics, 176 (1996), 357-64.

[8] H. Jenkins and J. Serrin, Variational problems of minimal surface type, II, Boundary value problems for the minimal surface equation, Arch. Rational Mech. Anal. 21 (1966), 321-42.

[9] F. Lopez, The classification of complete minimal surfaces with total curvature greater than $-12 \pi$, Trans. Aemr. Math. Soc. 334 (1992), 49-74.

[10] W. Meeks III and H. Rosenberg, The global theory of doubly periodic minimal surfaces, Invent. Math. 97 (1989), 351-79.

[11] W. Meeks III and H. Rosenberg, The geometry and conformal structure of properly embedded minimal surfaces of finite topology in $\mathbb{R}^{3}$, Invent. Math. 114 (1993), 625-39.

[12] W. Meeks III and B. White, Minimal surfaces bounded by convex curves in parallel planes, Comment. Math. Helvetici, 66 (1991), 263-78.

[13] J. C. C. Nitsche, On new results in the theory of minimal surfaces, Bull. Amer. Math. Soc. 71 (1965), 195-270.

[14] J. C. C. Nitsche, Lectures on Minimal Surfaces, Vol. 1, Cambridge University Press, 1989.

[15] R. Osserman, A Survey of Minimal Surfaces, Dover Publications, Inc. New York, 1986.

[16] R. Osserman and M. Schiffer, Doubly connected minimal surfaces, Arch. Ration. Mech. Anal. 58 (1974/75), 285-307.

[17] M. Shiffman, On surfaces of stationary area bounded by two circles, or convex curves, in parallel planes, Annals of Math. 63 (1956), 77-90. 
ReCeived SePtember 2, 1998.

AUSTRALIAN National UNIVERSITY

CANBerra, ACT 0200, AUSTRALIA

AND

ACAdEMIa Sinica

NANKang, TaIPEI, TaIwan 11529

REPUBLIC OF CHINA

E-mail addresses: yi@maths.anu.edu.au

MAJFHQccvax.sinica.edu.tw 